\title{
Cerebellar Processing Common to Delay and Trace Eyelid Conditioning
}

\author{
Hunter E. Halverson, ${ }^{1}{ }^{\circledR}$ Andrei Khilkevich, ${ }^{1}$ and ${ }^{\circledR}$ Michael D. Mauk ${ }^{1,2}$ \\ ${ }^{1}$ Center for Learning and Memory and ${ }^{2}$ Department of Neuroscience, University of Texas, Austin, Texas 78712
}

Results from previous lesion studies have been interpreted as evidence that the cerebellar cortex plays different roles for delay and trace conditioning of eyelid responses. However, the cerebellar cortex is organized by parasagittal stripes of Purkinje cells (PCs) that converge onto common deep nucleus neurons and receive common or related climbing fiber inputs. Based on this organization, we hypothesized that cerebellar tasks involving the same response system, such as delay and trace eyelid conditioning, would engage the same PCs and that the relationships between PC activity and expression of behavioral responses would be similar for both tasks. To test these hypotheses, we used tetrode recordings from eyelid PCs in rabbits during expression of delay- and trace-conditioned eyelid responses. Previous recording studies during delay conditioning described a strong relationship between eyelid PC activity and the kinematics of conditioned eyelid responses. The present results replicate these findings for delay conditioning and show that the same relationship exists during trace eyelid conditioning. During transitions from delay to trace responding, the relationship between eyelid PCs and behavioral responses was relatively stable. We found that an inverse firing rate model tuned to predict $\mathrm{PC}$ activity during one training paradigm could then predict equally well the $\mathrm{PC}$ activity during the other training paradigm. These results provide strong evidence that cerebellar cortex processing is similar for delay and trace eyelid conditioning and that the parasagittal organization of the cerebellum, not the conditioning paradigm, dictate which neurons are engaged to produce adaptively timed conditioned responses.

Key words: cerebellum; eyelid conditioning; modeling; Purkinje cell; tetrode recordings; trace conditioning

\section{Significance Statement}

A variety of evidence from eyelid conditioning and other cerebellar-dependent behaviors indicates that the cerebellar cortex is necessary for learning and proper timing of cerebellar learned responses. Debates exist about whether trace eyelid conditioning data show that fundamentally different mechanisms operate in the cerebellum during tasks when input from the forebrain is necessary for learning. We show here that learning-related changes in a specific population of Purkinje cells control the timing and amplitude of cerebellar responses the same way regardless of the inputs necessary to learn the task. Our results indicate the parasagittal organization of the cerebellar cortex, not the complexity of inputs to the cerebellum, determines which neurons are engaged in the learning and execution of cerebellar-mediated responses.

\section{Introduction}

Pavlovian eyelid conditioning has proven useful for analyses of neural mechanisms of learning and information processing in brain regions such as the hippocampus, cerebellum, and prefrontal cortex. Delay and trace eyelid conditioning differ only in the

\footnotetext{
Received Feb. 16, 2018; revised June 29, 2018; accepted July 6, 2018.

Author contributions: H.E.H. wrote the first draft of the paper; H.E.H., A.K., and M.D.M. edited the paper; H.E.H. and M.D.M. designed research; H.E.H. performed research; H.E.H. and A.K. analyzed data; H.E.H. and M.D.M. wrote the paper.

This work was supported by National Institute of Mental Health (Grants MH46904 and MH74006 to M.D.M). The authors declare no competing financial interests.

Correspondence should be addressed to Hunter E. Halverson, Center for Learning and Memory, University of Texas at Austin, 1 University Station, C7000, Austin, TX 78712. E-mail: hunter.halverson@utexas.edu. DOI:10.1523/JNEUROSCI.0430-18.2018

Copyright $\odot 2018$ the authors $\quad 0270-6474 / 18 / 387221-16 \$ 15.00 / 0$
}

relative timing of the stimuli: for delay conditioning, the conditioned stimulus (CS) and unconditioned stimulus (US) overlap in time, whereas trace conditioning involves a temporal gap between CS offset and US onset. Even so, there are important differences in the involvement of forebrain structures (Christian and Thompson, 2003). Although the cerebellum is necessary for both, lesions in various forebrain regions affect trace but not delay conditioning (Solomon and Moore, 1975; Berger and Orr, 1983; Woodruff-Pak et al., 1985; Solomon et al., 1986; Moyer et al., 1990; Kalmbach et al., 2009, 2010a).

Recent studies provide a straightforward mechanistic explanation for the forebrain dependence of trace eyelid conditioning. Using stimulation of mossy fibers as the CS, Kalmbach et al. (2010b) established that cerebellar learning requires that CSactivated mossy fiber inputs overlap in time with climbing fiber 
inputs activated by the US. Subsequently, Kalmbach et al. (2011) showed that two mossy fiber inputs, one lasting the duration of a CS and one persisting into the trace interval, were sufficient for the cerebellum to acquire responses with properties that quantitatively match trace eyelid responses with a traditional tone CS. Combined, these findings suggest that the necessary forebrain contribution is to provide the cerebellum with a mossy fiber input that persists into the trace interval to engage cerebellar learning (Clark and Squire, 1998; Siegel et al., 2012). Therefore, from the cerebellum's perspective, this persistent input transforms trace conditioning into delay conditioning such that common cerebellar mechanisms would operate in both paradigms (Kalmbach et al., 2010b, 2011; Siegel et al., 2012). This view is supported by studies showing that inactivation of cerebellar cortex produces parallel effects during delay and trace eyelid conditioning on the expression and timing of conditioned responses (CRs) (Kalmbach et al., 2010a).

This explanation is challenged by studies showing that cerebellar cortex lesions do not affect the acquisition or properly timed expression of trace eyelid responses and observations that different parts of the cerebellar cortex may mediate trace versus delay (Woodruff-Pak et al., 1985, 2006; Kishimoto et al., 2001a,b,c; Brown et al., 2010). In contrast, cerebellar cortex lesions prevent acquisition and affect the timing of already learned delay responses (Perrett et al., 1993; Perrett and Mauk, 1995; Garcia and Mauk, 1998; Garcia et al., 1999). These findings have been interpreted as evidence that cerebellar cortex contributions and mechanisms differ for delay and trace eyelid conditioning (Takehara-Nishiuchi, 2018). However, this view seems to contradict the parasagittal zone organization of cerebellar cortex, where zones control a specific response system (Eccles et al., 1967; Ekerot and Larson, 1979; Groenewegen et al., 1979). If correct, this view also requires new ways to think about cerebellar learning under varied circumstances.

Purkinje cells (PCs) provide the output of cerebellar cortex and previous studies established that learning-dependent decreases in eyelid PC activity control the kinematics of delay CRs (Halverson et al., 2015a). To address the conflicting views of cerebellar involvement in trace conditioning, we have recorded eyelid PC activity during delay and trace conditioning. We find that the relationship between eyelid CRs and PC single-unit activity share common properties across delay and trace conditioning. An inverse firing rate model revealed that the correspondence between learning-related activity in PCs and kinematic properties of eyelid CRs did not differ for delay and trace conditioning. Instances where the same PC was recorded during the transition from delay to trace conditioning showed that the PCs relate to the behavioral responses similarly for delay and trace conditioning. These data support the hypothesis that trace and delay eyelid conditioning engage the same PCs and that the cerebellar cortex operates under similar rules for delay and trace eyelid conditioning.

\section{Materials and Methods}

\section{Subjects}

The subjects were three male New Zealand albino rabbits (Oryctolagus cuniculus; Myrtle's Rabbitry) weighing 2-3 $\mathrm{kg}$ at experiment onset. Treatment of rabbits and surgical procedures were in accordance with National Institutes of Health guidelines and an institutionally approved animal welfare protocol. All rabbits were maintained on a $12 \mathrm{~h} \mathrm{light/dark}$ cycle.

\section{Surgery}

One week before the start of recording, rabbits were removed from the home cage and anesthetized with a mixture of acepromazine $(1.5 \mathrm{mg} / \mathrm{kg})$ and ketamine ( $45 \mathrm{mg} / \mathrm{kg})$. After onset of anesthesia, animals were placed in a stereotaxic frame and maintained on isoflurane $(1 \sim 2 \%$ mixed in oxygen) for the remainder of the surgery. Under sterile conditions, the skull was exposed with a midline incision $(\sim 5 \mathrm{~cm})$ and four holes were drilled for screws that anchored the head bolt in place. The animal's head was then positioned with lambda $1.5 \mathrm{~mm}$ ventral to bregma and a craniotomy was drilled out at $5.9 \mathrm{~mm}$ posterior and $6.0 \mathrm{~mm}$ lateral to lambda for cerebellar cortex. The skull surface was marked and skull fragments were carefully removed from the craniotomy along with the dura matter under visual guidance. A custom-made hyperdrive array (12 or 16 tetrodes, two references) fitted with an electronic interface board (EIB-54 or EIB-36-16TT; Neuralynx) was implanted in the left anterior lobe of the cerebellar cortex at $17.8 \mathrm{~mm}$ ventral to lambda. Final ventral placement of tetrodes during surgery was between 1 and $2 \mathrm{~mm}$ above the target coordinate to allow advancement of the tetrodes to the target. The cortex hyperdrives were positioned at a $40^{\circ}$ angle caudal to vertical to avoid the cerebellar tentorium. This region of the cerebellum has been shown to be involved in acquisition and expression of well timed eyelid CRs in rabbits (Garcia et al., 1999; Green and Steinmetz, 2005; Ohyama et al., 2006; Kalmbach et al., 2010a; Halverson et al., 2015a). The bundle cannula of the hyperdrive was lowered to the surface of the brain and the craniotomy was sealed with low-viscosity silicon (Kwik-Sil; World Precision Instruments). A screw attached to an insulated silver grounding wire (0.003 inches bare, 0.0055 inches coated; A-M Systems) was then screwed into the skull. The silver wire was also attached to the ground channel of the EIB with a gold pin. The head bolt, screws, and hyperdrive were secured with dental acrylic (Bosworth Fastray, Pink; Harry J. Bosworth Company) and the skin was sutured where the skull and muscle were exposed. Finally, two stainless steel stimulating electrodes were implanted subdermally caudal and rostral to the left eye. Rabbits were given analgesics and antibiotics for $2 \mathrm{~d}$ after surgery and monitored until fully recovered.

\section{Recording and unit isolation}

Each independently movable tetrode was composed of four nichrome wires (12 $\mu \mathrm{m}$ diameter; Kanthal Palm Coast) twisted and partially melted together to form a tetrode. Individual wires of each tetrode were connected to the EIB with gold pins and all four wires of the reference tetrodes were connected to a single reference channel of the EIB. Each tetrode was gold plated to reduce final impedance between 0.5 and $1.5 \mathrm{M} \Omega(1.0 \mathrm{M} \Omega$ targeted) measured at $1 \mathrm{kHz}$ (impedance tester IMP-1; Bak Electronics). Tetrodes were placed over the left anterior lobe and advanced to within $2.0 \mathrm{~mm}$ of the target during surgery using stereotaxic guidance. Tetrodes were then lowered in $40-80 \mu \mathrm{m}$ increments during turning sessions $(\sim 1 \mathrm{~h})$ until at least one stable single unit was identified; there were often multiple units on a single tetrode. After turning sessions, tetrodes were allowed to stabilize for at least $24 \mathrm{~h}$ and units were checked again before recording and behavioral training commenced. Putative PCs were initially identified by their higher baseline firing rate relative to other cerebellar cortical interneurons and later confirmed by identifying complex spikes during cluster cutting (Fig. $1 B, C$ ) (Halverson et al., 2015a). Recordings were done once a single unit was identified and stable without knowledge of the origin of its climbing fiber input. Single units were initially isolated from the multiunit recording based on consistent differences in peak amplitudes of the waveforms across the four channels of the tetrode (Fig. 1B). Putative PCs were then examined using the late peak or late valley feature of the waveforms. Complex spikes separated into an additional cluster in these late parameters, which then formed two clusters from the original one isolated using peak: one containing the simple spikes and the other the complex spikes (Figure $1 B, C$ ). All PC recordings demonstrated spontaneous complex spikes that were verified with a complex spike-triggered average of simple spikes to confirm the characteristic 20-30 ms pause in simple spikes after a complex spike (Halverson et al., 2015a). Eyelid PCs were identified by the presence of short-latency ( $8-15 \mathrm{~ms}$ after US onset) US-evoked complex spikes in the peristimulus histogram/raster plot and continuous multiunit recording (Fig. $1 E, F$ ). The stimulus artifact from the US may have obscured our ability to identify complex spikes specifically during the stimulus pulses spaced at 10-12 ms intervals after US onset. This could partially inhibit 


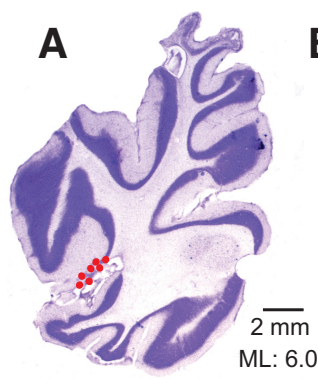

B

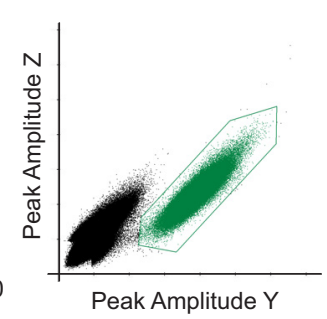

CS

$\mathbf{F}$
C

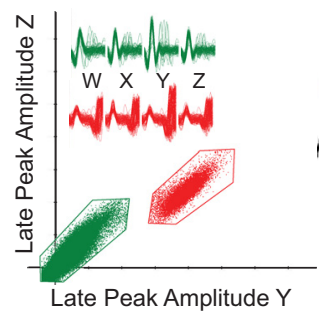

D

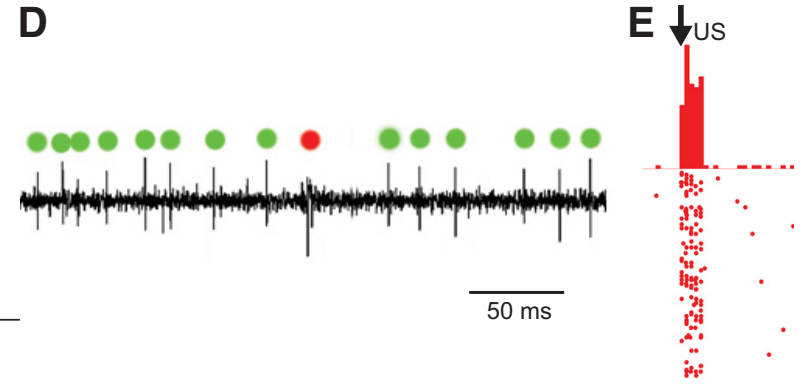

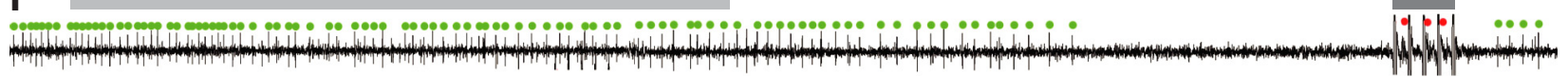

US

Figure 1. Recordings from cerebellar cortex and classification of eyelid PCS.A, Sagittal section of cerebellar cortex showing the final location (red dots) of tetrodes that recorded eyelid PC activity. The majority of eyelid PC recordings were taken in close proximity $(240 \mu \mathrm{m})$, which produced overlap in the indicated locations. $\boldsymbol{B}$, Example cluster cutting window plotted as peak to peak showing a single unit (green) isolated from the multiunit recording (black). C, Isolation of complex spikes (red) and simple spikes (green) from the original cluster cut in $\boldsymbol{B}$ using the late peak parameter. The color-coded waveforms on the four channels of the tetrode are also shown for each cluster. $\boldsymbol{D}$, Multiunit continuous recording from a single wire of a tetrode showing the simple (green) and complex (red) spikes from a PC identified with the cluster cutting method in $\boldsymbol{B}$ and $\boldsymbol{C}$. $\boldsymbol{E}$, Peristimulus histogram (10 ms bins) and raster plot of the complex spikes from an eyelid PC recording during an eyelid conditioning session. The raster plot is arranged sequentially with the first trial on the bottom. This recording was classified as an eyelid PC based on the identified complex spikes being elicited by the eyelid stimulation US (arrow). $\boldsymbol{F}$, Multiunit continuous recording from a single wire of a tetrode showing simple (green dots) and complex (red dots) spikes from an isolated eyelid PC during a single trace conditioning trial when the rabbit showed a CR. The gray line represents the $500 \mathrm{~ms}$ tone $C S$; dark gray line represents the $50 \mathrm{~ms}$ US.

our ability to identify all US-elicited complex spikes, but would not prevent classifying a PC as eyelid or not because the US would often evoke a burst of three or more complex spikes on a paired trial. PC single units that did not pass these confirming steps for placement in the eyelid region of the cerebellar cortex were designated as non-eyelid recordings and were not analyzed further. All PCs included in the analysis had confirmed spontaneous complex spikes (Fig. 1D) and all units were held throughout the entire session. Any recording that was lost during a recording session was not included in the analysis.

Neuronal signals were first preamplified at unity gain. The signals were then fit to a window between 250 and $2000 \mu \mathrm{V}$ and band-pass filtered (0.3-6 kHz; Neuralynx). Neural signals that exceeded a channel amplitude threshold were digitized and stored at $32 \mathrm{kHz}$ (Cheetah system; Neuralynx). Custom interactive cluster cutting programs were used to isolate and identify single units. Waveform characteristics were plotted as a $2 \mathrm{D}$ scatter plot of one wire of the tetrode versus another in terms of energy, peak, and valley measures. The energy measure represents the square root of the sum of the squared points for the entire waveform. The peak measure is the maximum height (positive amplitude) of the waveform. The valley measure is the maximum depth (negative amplitude) of the waveform. For recordings in cerebellar cortex, late peak and valley features, defined as the maximum peak or valley during the last five points of the 32 points that make up each waveform, were also used to identify the later component of the complex spikes from the earlier peak of the simple spikes.

\section{Conditioning procedure}

Conditioning experiments and recordings were done in custom training chambers $(89 \times 64 \times 49 \mathrm{~cm})$. Rabbits were placed in a plastic restrainer and the ears were stretched over a foam pad and taped down to limit head movement. An adjustable infrared emitter/detector was secured in place with the head bolt and aligned to the middle of the left eye. The infrared emitter/detector measured upper eyelid position by converting the amount of emitted infrared light reflected back to the detector, which increases as the eyelid closes, to a voltage. The signal was amplified to yield a signal that was linearly related to upper eyelid position $( \pm 0.1$ $\mathrm{mm}$ ). The eyelid position detector was then calibrated before each training session by delivering a test US to elicit maximum eyelid closure $(6.0$ $\mathrm{mm})$. The corresponding voltage deflection $(\sim 6 \mathrm{~V})$ was then divided by $6 \mathrm{~mm}$ to obtain a $\mathrm{mm} / \mathrm{V}$ calibration. Each training chamber was also equipped with a speaker connected to a stereo equalizer and receiver, which were connected to a computer that generated the tone CS. The CS used during training was either a 1 or $9.5 \mathrm{kHz}$ sinusoidal tone $(85 \mathrm{~dB})$, which ramped at onset and offset with a $5 \mathrm{~ms}$ time constant to avoid audible clicks from the speaker. To deliver the US, leads from a stimulus isolator (Model 2100; A-M Systems) were attached to electrodes caudal and rostral to the eye. The US was eyelid stimulation, which consisted of trains of $1 \mathrm{~ms}$ current pulses delivered at $100 \mathrm{~Hz}$ for $50 \mathrm{~ms}$ with the intensity adjusted for each animal to be just above threshold to elicit a full eyelid closure (between 0.8 and $1.5 \mathrm{~mA}$, depending on the condition of the implanted wires).

Stimulus presentation was controlled by custom software operated on a Windows XP-based computer. To permit temporal alignment of neural and behavioral responses, digital timing pulses were generated by the computer controlling stimuli and measuring behavior and were sent to the digital input port on the Digital Lynx acquisition system (Neuralynx). During initial paired delay conditioning, the tone CS was $550 \mathrm{~ms}$, which coterminated with the 50 ms eyelid stimulation US, which produced an interstimulus interval (ISI) of $500 \mathrm{~ms}$. All rabbits were initially trained with delay conditioning at ISI 500. Trace conditioning sessions consisted of the same $1 \mathrm{kHz}$ tone presented for $500 \mathrm{~ms}$ with a $500 \mathrm{~ms}$ stimulus free trace interval ending with the same $50 \mathrm{~ms}$ eyelid stimulation US. The same frequency tone CS was used intentionally for both delay and trace conditioning to determine how eyelid PCs would respond during the transition from delay to trace conditioning and also to confirm if the timing of the learning-related changes would also shift as trace CRs emerged. Two rabbits were also trained at ISI 1000 with a $9.5 \mathrm{kHz}$ tone CS and then switched to trace conditioning with the $1 \mathrm{kHz}$ tone to examine eyelid PC responses during CRs trained at the same interval. Each training session consisted of 12 nine-trial blocks (108 trials), with each block starting with a CS-alone trial followed by eight paired CS-US trials. The mean intertrial interval was $30 \mathrm{~s}$ with a range of $20-40 \mathrm{~s}$.

\section{Data analysis}

After cluster cutting, all subsequent data analysis was performed using custom-written scripts in MATLAB (The MathWorks).

Eyelid data analysis. For each trial, $2500 \mathrm{~ms}$ of eyelid position $(200 \mathrm{~ms}$ pre-CS, $2300 \mathrm{~ms}$ post-CS) were collected at $1 \mathrm{kHz}$ and at 12 bit resolution. Data were stored to a computer disk for subsequent offline analysis. Eyelid position data were passed through a low-pass filter. Response measures calculated for each trial included CR amplitude, latency to CR criterion, and latency to $\mathrm{CR}$ onset. CR amplitude was defined as the value of eyelid position from the baseline at the time of US onset. Latency to CR criterion was defined as the time point at which the $\mathrm{CR}$ reached the $0.3 \mathrm{~mm}$ criterion to be designated as a CR. Latency to CR onset was determined using a custom-written two-step algorithm. The first step was designed to detect the initial deflection away from the pre-CS baseline; the second step used linear interpolation to determine the exact time 
of CR onset. For further analyses, eyelid trajectories were truncated at US onset to exclude noncerebellar influence on the eyelid movement.

Single-unit data analysis. Instantaneous firing rate of each single-unit recording was estimated on every trial using a one-sided Gaussian kernel with a $25 \mathrm{~ms} \mathrm{SD}$ window. We chose to use a one-sided Gaussian to prevent neural responses related to the US from contaminating unit activity during the CS in delay conditioning or trace interval in trace conditioning. For every PC unit, the firing rate was normalized by the value of the baseline firing rate during $1500 \mathrm{~ms}$ of pre CS activity.

Linear inverse model analysis. We applied a linear inverse model approach to study the relationship between eyelid PC simple spike firing rate and CR kinematics during delay and trace training sessions. The linear model was constructed in the following way:

$$
r(t)=a \cdot y(t+\Delta t)+b \cdot \dot{y}+c \cdot \ddot{y}(t+\Delta t)+r_{c}
$$

Where $y(t), \dot{y}(t)$, and $\ddot{y}(t)$ are eyelid position, velocity and acceleration, respectively, as a function of time, $r(t)$ is eyelid PC firing rate, $\Delta t$ is a time lag, $a-c$ are linear regression coefficients, and $r_{\mathrm{c}}$ is the baseline firing rate value before the CS. The analysis described below was done only on trials with CRs present. For every session, $75 \%$ of CR trials were used for the regression procedure to find the linear coefficients $a-c$, also referred to as position, velocity, and acceleration weights. The fitting was repeated for the allowed range of $\Delta t$, the lag time, along with the corresponding linear coefficients that produced the best fit were chosen. Then, to avoid overfitting, a separate $25 \%$ of trials were used to test the accuracy of the prediction using parameters estimated from the first 75\% of CR trials. The process was repeated four times, each time shifting which trials were used as fit data and test datasets. Both fitting and prediction parts were done using eyelid position, velocity, acceleration, and PC firing rate time profiles averaged through the corresponding fit or test trials. Before fitting, average eyelid position, velocity, and acceleration profiles were normalized to have the difference between maximum and minimum values equal to one, this made it possible to compare their weights directly. Accuracy of the inverse model prediction was measured only on test trials. Reported $R^{2}$ values and weights are averages across the four iterations unless noted otherwise. Fitting procedure was performed using the lsqnonlin MATLAB function.

Tracking individual eyelid PCs for consecutive days. There were instances where the pattern of activity on an individual tetrode provided evidence that the same PC single unit was being recorded for a number of consecutive days (sessions). Single units were putatively classified as the same unit on consecutive days if all of the following criteria were met: the recordings came from the same tetrode, the tetrode was not moved between days, the cluster patterns (and waveforms) were similar across days, and the ISI histograms were consistent across all days of the recordings for a given single unit. Attempting to track single units was done intentionally to investigate how individual eyelid PCs responded during both delay and trace eyelid conditioning.

Grouping. To further investigate how eyelid PCs correspond to the onset and amplitude of eyelid CRs, we performed a grouping procedure. Trials with CRs from sessions with the same paradigm were combined and divided into two or three equal subgroups of trials according to CR onset or amplitude. Because trials with late CR onset are more likely to have lower than average CR amplitude, we performed an additional step to disassociate between variance in CR onset and amplitude. Specifically, the analyses insured that the mean values of the unrelated parameter (CR amplitude for grouping by CR onset analyses) are within a $10 \%$ difference across all groups of CRs. For that, after initial grouping of trials based on CR variance, we iteratively removed $5 \%$ of trials in the group that had the largest difference from the target parameter (e.g., for grouping by $\mathrm{CR}$ onset, the group with the smallest mean CR amplitude will remove $5 \%$ of trials with the smallest CRs and the group with largest mean CR amplitude will remove $5 \%$ of the trials with the largest CRs). This procedure was repeated iteratively until differences between the mean values of the parameter unrelated to the grouping were within $10 \%$ across groups. For all grouping analyses, the maximum fraction of discarded trails was within $20 \%$. The number of trials in the non-CR group was not controlled and was typically low because subjects were well trained. Subgroup behavioral and neural data were then averaged within each group and superimposed for each training paradigm. The absence of overlap in $95 \%$ confidence intervals between groups of average behavioral responses and eyelid PC single-unit activity were used as evidence of reliable separation.

\section{Histology}

After the conclusion of experiments, the final tetrode placement was determined by making small marking lesions by passing $10 \mu \mathrm{A}$ of anodal DC current for $10 \mathrm{~s}$ through tetrodes that yielded data. Animals were killed with an overdose of sodium pentobarbital and perfused intracardially with $0.9 \%$ saline $(\sim 1.0 \mathrm{~L})$, followed by $10 \%$ formalin $(\sim 1.0 \mathrm{~L})$. Heads were postfixed in formalin for at least $3 \mathrm{~d}$, after which tetrodes were removed and the brains were extracted. Brains were then cryoprotected in $30 \%$ sucrose in formalin for $3 \mathrm{~d}$, embedded in an albumin gelatin mixture, and the cerebellum was sectioned using a freezing microtome at $40 \mu \mathrm{m}$. Tissue was mounted on slides and stained with cresyl violet. Sections were then examined to determine the final location of each tetrode and this depth was compared with depth records from turning sessions to identify the location of unit recordings (Fig. 1A).

\section{Results}

\section{Location and identification of eyelid PCs}

We recorded a total of 493 units that were identified as PCs on the basis of their spiking activity and presence of complex spikes. Using criteria described in the Materials and Methods, 46 of these units were classified as eyelid PCs (see also Halverson et al., 2015a). These eyelid PCs were recorded on 20 of the 40 total tetrodes implanted in the three rabbits. The calculated location of each of the 46 recordings is indicated by the red dots in Figure $1 \mathrm{~A}$. Many dots overlap because of the tight clustering of the recording locations. These sites were clustered near the primary fissure in deeper parts of lobules HVI and HV of the anterior lobe, often in the sulcus that extends most caudally toward the deep nuclei.

\section{Eyelid PC responses during delay and trace conditioning}

If the cerebellar cortex operates similarly for delay and trace eyelid conditioning, then we would expect that the relationship between PC responses and eyelid CRs in the two paradigms should also be similar. Even though previous studies characterized this relationship for delay CRs (Halverson et al., 2015a), we recorded PCs during both delay and trace conditioning for the following reasons: (1) to make within-subject comparisons of $\mathrm{PC}$ responses during expression of delay and trace CRs, (2) to investigate PC responses during the transition from delay to trace responding, and, when possible, (3) to make within-PC comparisons between delay and trace conditioning when a single cell was held through enough sessions.

Each rabbit was initially trained using delay conditioning sessions with a $550 \mathrm{~ms}$ tone CS paired with a coterminating $50 \mathrm{~ms}$ eyelid stimulation US, yielding an ISI of $500 \mathrm{~ms}$, which, for convenience, we will refer to as Delay 500. Delay 500 training continued until each rabbit achieved a high rate of conditioned responding (above $80 \%$ CRs during a session) and until at least one eyelid PC(s) was encountered (Fig. 2A, bottom). After Delay 500 conditioning and on a session where at least one eyelid PC was present, training was switched to trace conditioning (Trace 500/500, indicating a $500 \mathrm{~ms}$ tone and $500 \mathrm{~ms}$ trace interval). With additional trace conditioning sessions, robust expression of trace eyelid CRs was acquired. Trace 500/500 training sessions continued to record eyelid PCs during robust expression of trace responses.

In two rabbits, training was then shifted back to delay conditioning using an ISI of $1000 \mathrm{~ms}$ (Delay 1000) and these sessions continued until eyelid PCs were encountered. Delay 1000 allowed for 
A

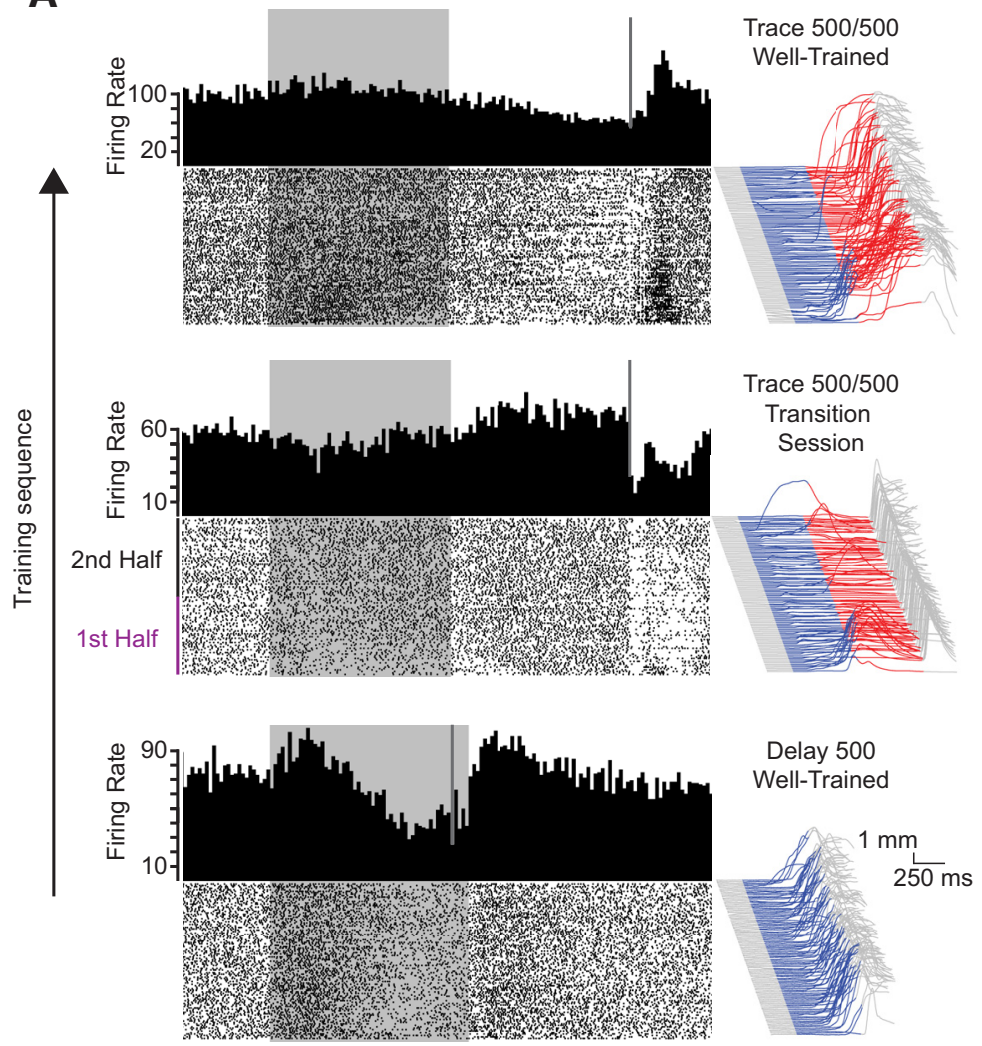

C

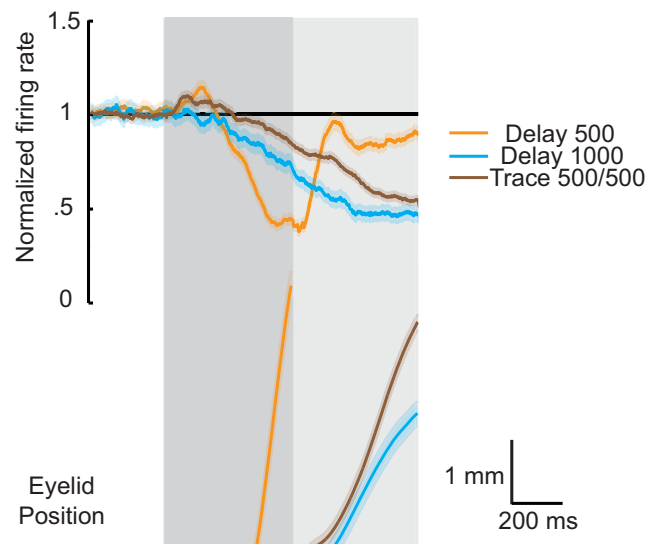

Eyelid Velocity

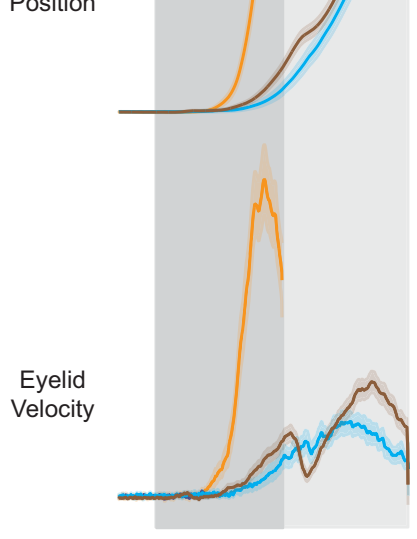

$$
200 \mathrm{~ms}
$$

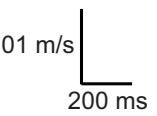

B

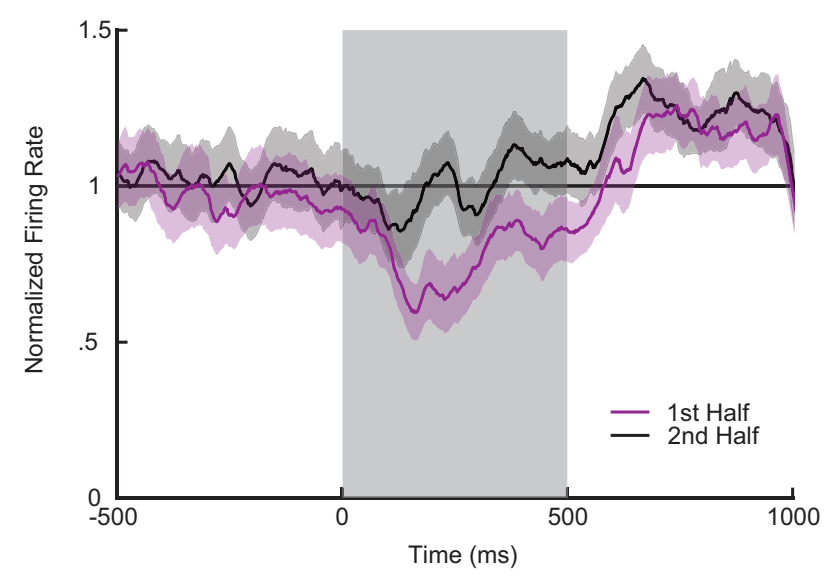

Figure 2. A, Examples of eyelid (Rs and eyelid PC recordings during the transition from well trained at Delay 500 (bottom) to the transition session of Trace $500 / 500$ (middle) and well trained at Trace 500/500 (top). The bottom example shows the decrease in PC activity associated with expression of well trained CRs during Delay 500. The transition session shows the decrease in PC activity and CRs associated with the Delay $500 \mathrm{ISI}$ extinguish during the first half of the session. The top example shows how the decrease in PC activity shifts into the trace interval to match expression of well trained CRs during Trace 500/500. In the peristimulus histograms ( $10 \mathrm{~ms}$ bins) the duration of the tone CS is represented with the gray box and the onset of the eyelid stimulation US is represented by the dark gray line for this and all subsequent figures. For the behavioral sweeps, blue represents the duration of the tone stimulus and red represents the trace interval. Behavioral sweeps are aligned trial to trial with the raster plots in each example. $\boldsymbol{B}$, Instantaneous firing rate (normalized to baseline) of the example PC during the transition session from $\boldsymbol{A}$. Extinction of the decrease in $\mathrm{PC}$ activity related to the expression of the previously trained Delay 500 CRs occurs in the first half of the session (purple trace) during the CS period (gray box). $\mathrm{PC}$ activity returns to baseline levels after (Rs have extinguished in the second half of the session (black trace). C, Average normalized eyelid PC firing rate and kinematic variables of the eyelid (Rs during Delay 500 (orange), Delay 1000 (cyan), and Trace 500/500 (brown). For this and all subsequent figures, the shading around the average lines represents 95\% confidence intervals for each kinematic variable and eyelid PC activity. The dark gray bars represent duration of the CS and the light gray bars represent the trace interval.

comparisons between eyelid CRs and eyelid PC activity trained to the same overall interval (1000 ms) during delay and trace conditioning. Then, as before, training was switched back to Trace 500/ 500 , now using a different tone CS frequency to further differentiate between the two training paradigms and to examine single PC responses to different inputs. These rabbits were then given several more Trace 500/500 conditioning sessions to record a sufficient number of eyelid PCs to make comparisons of their responses during asymptotic performance of Trace 500/500 and Delay 1000 CRs.

\section{Relationship between eyelid PC activity and conditioned eyelid responses}

On the transition session from Delay 500 to Trace 500/500, delay conditioned eyelid responses and the decreases in PC activity 
associated with them were both extinguished by the halfway point of the session (Fig. $2 A$, middle raster plot). Figure $2 B$ shows average eyelid PC firing rate for the first half of the transition session where CRs are present versus the second half of the same session where Delay 500 CRs have extinguished. As Trace 500/ 500 CRs emerged in later sessions, the timing of the CRs and the associated decreases in eyelid PC activity both shifted later, usually beginning in the trace interval (Fig. $2 A$, top), which is characteristic of eyelid CRs with trace conditioning.

Next, we used session averages of eyelid PC firing rate to make group comparisons of eyelid PC responses for Delay 500, Trace 500/500, and Delay 1000. Average instantaneous firing rate of eyelid PCs normalized to the pre-CS baseline, along with position and velocity of eyelid CRs for each training paradigm, are shown in Figure $2 C$. The superimposed averages indicate differences in the responses of eyelid PCs between paradigms trained at different intervals closely track the timing of eyelid CRs for Delay 500, Trace 500/500, and Delay 1000. The shaded regions around the average lines in Figure 2C represent the 95\% confidence intervals for each measure. These results from average PC and eyelid kinematic data are generally consistent with the idea that eyelid PCs are controlling the timing and expression of eyelid CRs during both delay and trace conditioning. Subsequent analyses described below sought to quantify this further.

\section{Control of CR onset by eyelid PC activity}

Eyelid CRs display trial-to-trial variability in the timing of response onset, which not surprisingly increases as the ISI increases. This variability introduces a degree of averaging artifact that can obscure evaluation of how precise the trial-to-trial relationship is between eyelid PC responses and expression of eyelid CRs. This trial-to-trial relationship is illustrated visually in Figure $3, A$ and $B$, which shows an example eyelid $\mathrm{PC}$ with the raster plot and behavior with trials shown in chronological order (Fig. $3 A$ ) and again sorted by CR onset latency (Fig. 3B). The red dots in each row of the raster plots indicate the latency to onset of the CR on each trial. This trial-to-trial relationship between decreases in individual eyelid PC activity and trace CR onset appears to be similar to that demonstrated previously for delay conditioning (Halverson et al., 2015a).

As one approach to overcome this averaging artifact and to quantify the relationship between eyelid PC responses and eyelid CRs, we grouped trials for each training paradigm in separate groups based on the time of CR onset. Ranges of CR onsets were chosen to produce three groups (two groups from Delay 1000 because of smaller number of recorded eyelid PCs) with approximately equal numbers of trials. Because CRs with later onset times are more likely to have smaller amplitude, we added an additional condition to the grouping procedure, which ensured that the mean values of CR amplitudes were within $10 \%$ difference across groups. The fourth group containing non-CR trials represented a smaller dataset as there were usually few non-CR trials in these sessions. Figure 3, $C-E$, shows, separately for the three training paradigms the grouped behavioral and neural data superimposed. For Delay 500, Delay 1000, and Trace 500/500 conditioning paradigms, eyelid PC activity reliably tracked the differences between CR onsets. Non-overlap of the 95\% confidence intervals of average PC firing rate for each group indicated significant differences between the timing of decreases in PC activity controlling CRs, with different average onset latencies for all three conditioning paradigms. In all cases, there was little to no change in eyelid PC activity for non-CR groups (blue traces). The significant differences in eyelid PC responses between CRs of different timing are consistent with the hypothesis that eyelid PC activity largely determines the presence and timing of eyelid CRs for both delay and trace conditioning.

Aligning eyelid PC and behavioral responses to CR onset provides a more direct assessment of how eyelid PC activity relates to CR timing. Figure 3, $G$ and $H$, shows superimposed average (Fig. $3 G$, normalized to baseline; Fig. $3 H$, average firing rate) eyelid $\mathrm{PC}$ responses for all three training paradigms. In each case, eyelid PC activity decreases to the same level relative to baseline before $\mathrm{CR}$ onset (Fig. 3G,H). When aligned to the onset time, CR profiles for Delay 500 and Trace 500/500 show considerable overlap (Fig. $3 F$ ). Similarly, the average eyelid PC activity for these two forms of training are also almost indistinguishable (Fig. $3 G$ ). In both instances, there is a small increase in activity, followed by a decrease that precedes CR onset. Delay 1000 CRs have a slower rise time after CR onset and, similarly, there is a slower decrease in eyelid PC activity before onset. The pre-CR increase in activity is also absent, perhaps reflecting that this increase is associated with CRs with faster rise times. Despite the subtle differences between Delay 1000 and the other two paradigms, the one feature shared by all three types of responses is that PC activity falls to $\sim 80 \%$ of the pre-CR level at the time of CR onset. Indeed, the overlap in the $95 \%$ confidence intervals for all three response types is consistent with no significant differences between groups (Fig. $3 G, H)$. The above data demonstrate strong similarities between delay and trace conditioning in the relationship between average eyelid PC activity and the timing of CRs. Changes in eyelid PC activity also directly relate to CR amplitude during delay conditioning (Halverson et al., 2015a), which we explore next.

\section{Control of CR amplitude by eyelid PC activity}

To investigate the relationship between eyelid PCs and CR amplitude, we used the same grouping method used for CR onset. Figure 4, $A-C$, shows average profiles of eyelid CRs grouped by CR amplitude, with the corresponding average eyelid PC activity for each training paradigm. Here, the analyses also ensured that the mean values of CR onset times were within 10\% difference across groups. Similar to the CR onset analysis, the grouped PC activity within each of the different training paradigms showed non-overlap in the $95 \%$ confidence intervals, indicating significant differences in average PC activity related to CRs with different amplitudes. In addition to the averaged grouped analysis, we also evaluated trial-by-trial relationships between eyelid PC activity and CRs to detect possible differences between delay and trace conditioning that analysis of averaged activity might obscure. We focused on eyelid PCs recorded during expression of CRs during Delay 1000 and Trace 500/500 sessions to equate for ISI across groups. The trial-by-trial relationship between CR amplitude and eyelid PC activity for two examples is shown in Figure $4 D$ (black $=$ Delay 1000 , red $=$ Trace 500/500). For both Delay 1000 and Trace 500/500, the spike counts for these PCs showed a strong linear relationship with CR amplitude $(r=-0.65$ for Delay 1000 and $r=-0.63$ for Trace 500/500). Analysis was done on the spike count during the last $500 \mathrm{~ms}$ of the ISI (normalized to the spike count $1000 \mathrm{~ms}$ before CS onset) versus CR amplitude on that trial. Points clustered near the $y$-axis represent non-CR trials of approximately zero amplitude. Figure $4 E$ shows a summary for every eyelid PC recording for Delay 1000 (black dots) and Trace 500/500 (red dots). The relationship between eyelid PC activity and CR amplitude was indistinguishable between Delay 1000 and Trace 500/500. Average Pearson correlation coefficients between eyelid PC spike count and CR amplitude were similar for both Delay $1000(r=-0.63 \pm 0.09)$ and Trace 500/500 $(r=$ 
A

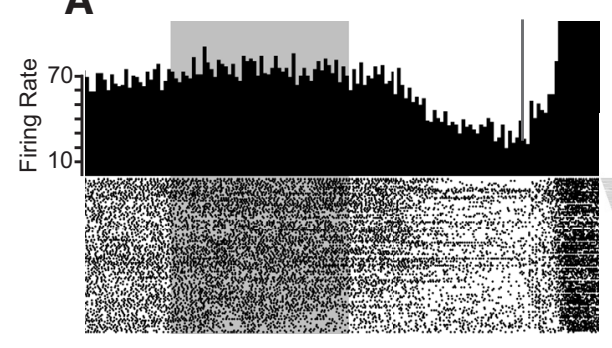

B

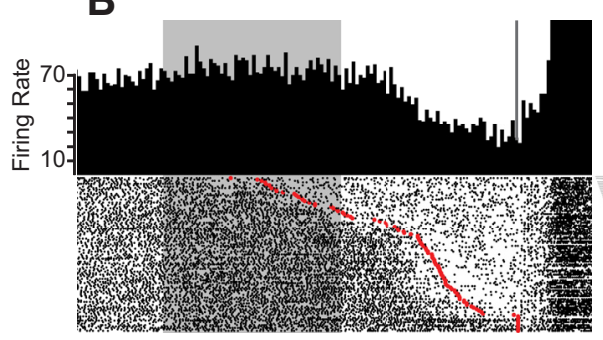

Sorted by CR onset

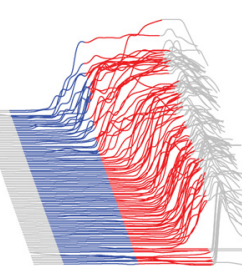

Delay 500

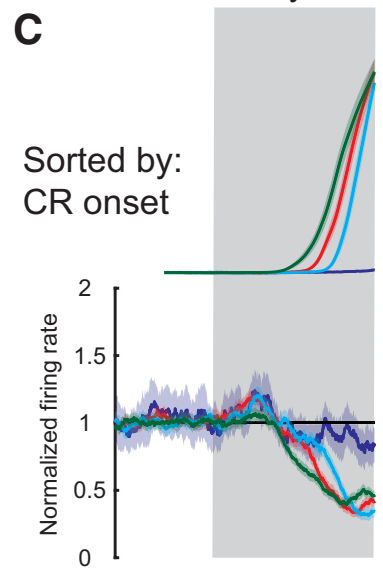

F

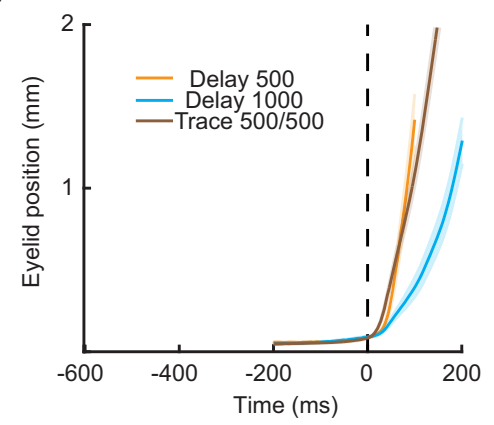

In order of the session $1 \mathrm{~mm}$

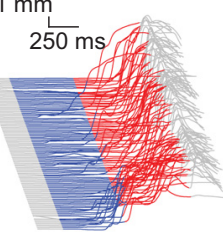

Trace 500/500

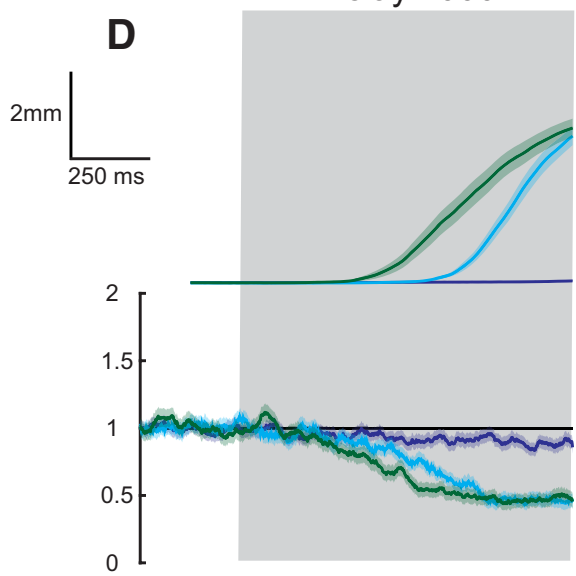

G

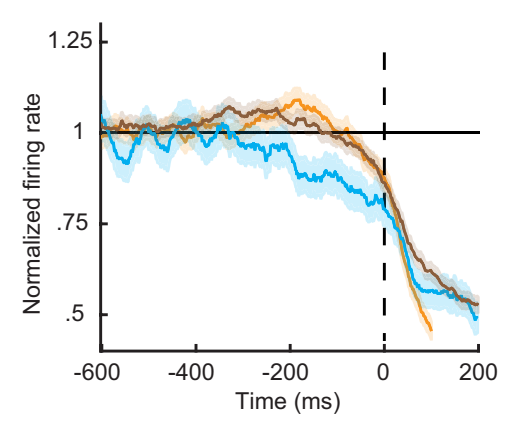

E

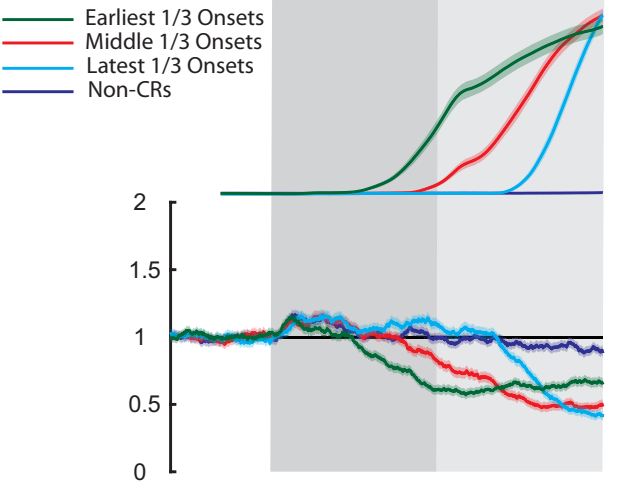

H

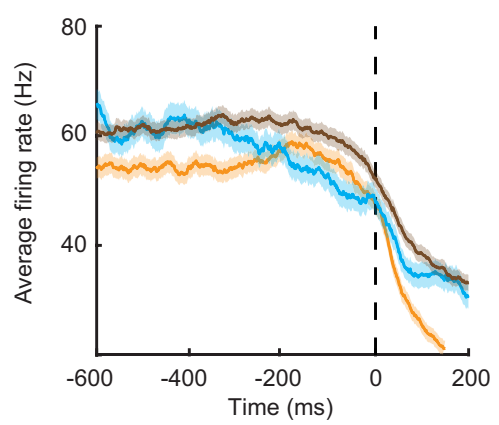

Figure 3. Peristimulus histograms and raster plots showing the close relationship between CR onset and decreases in the activity of an eyelid PC. Trials in the raster plot and behavior are in sequential order in $\boldsymbol{A}$ and sorted by CR onset latency (red dots) in $\boldsymbol{B}$. The same example sorted by latency to onset of the behavioral CRs is shown to illustrate the trial-to-trial relationship between $\mathrm{PC}$ activity and CRs. For the histograms and raster plots gray bars represent tone stimulus duration, darker gray line represents onset of eyelid stimulation. For the behavioral sweeps, blue represents the tone stimulus duration and red represents the trace interval. C $E$, Behavioral CRs and eyelid PC firing rate profiles sorted into groups according to CR onset and averaged within each ISI. CRs (top) and eyelid PC data (bottom) sorted by CR onset (green = earliest onset, red = middle onset, cyan = latest onset, blue = non-CRs). The dark gray bars represent duration of the CS and light gray represents the trace interval. $\boldsymbol{F}, \boldsymbol{G}$, Average eyelid $\mathrm{CRs}(\boldsymbol{F})$ and normalized instantaneous firing rate for all eyelid $\mathrm{PCs}(\boldsymbol{G})$ superimposed and aligned to $\mathrm{CR}$ onset (time $=0$, dotted line) recorded during Delay 500 (orange), Delay 1000 (cyan), and Trace 500/500 (brown). H, Same data shown in $\mathbf{G}$ represented as average firing rate. Eyelid PCs decrease activity by the same amount relative to CR onset during delay and trace conditioning.

$-0.62 \pm 0.04)$ and not significantly different between paradigms (two sample bootstrap test, $p=0.61,10,000$ repetitions) (Fig. $4 F)$. The mean values of linear regression slopes were also similar between paradigms $(-0.1 \pm 0.02$ for Delay $1000,-0.081 \pm 0.007$ for Trace 500/500) and not significantly different (two-sample bootstrap test, $p=0.34,10,000$ repetitions) (Fig. $4 G$ ). These data show that, at least in terms of CR amplitude, the similarities between eyelid PC responding in delay versus trace conditioning are not the result of averaging artifacts.

Tracking the same neuron from delay to trace conditioning In two instances, we were able to follow the same eyelid PC from delay conditioning through the later sessions of trace conditioning, where trace responding was at asymptote. The criteria for identifying a PC as being the same across sessions was independent of its trial-related responses and were based instead on the across-sessions consistency of the relative cluster location, waveform shape across the four tetrode channels, and ISI histograms. Recordings from the same tetrode were likely from the same PC across sessions in the cases where each of these variables were consistent.

One example involved an eyelid PC that was followed from expression of delay conditioning (Delay 500 well trained; Fig. 5, bottom) through Trace 500/500 well timed expression of CRs (Fig. 5, top). Peristimulus histograms, raster plots, and behavior are shown for five sessions as the rabbit transitioned from delay to trace conditioning. The presence or absence of CRs and their timing was always accompanied by parallel changes in the re- 

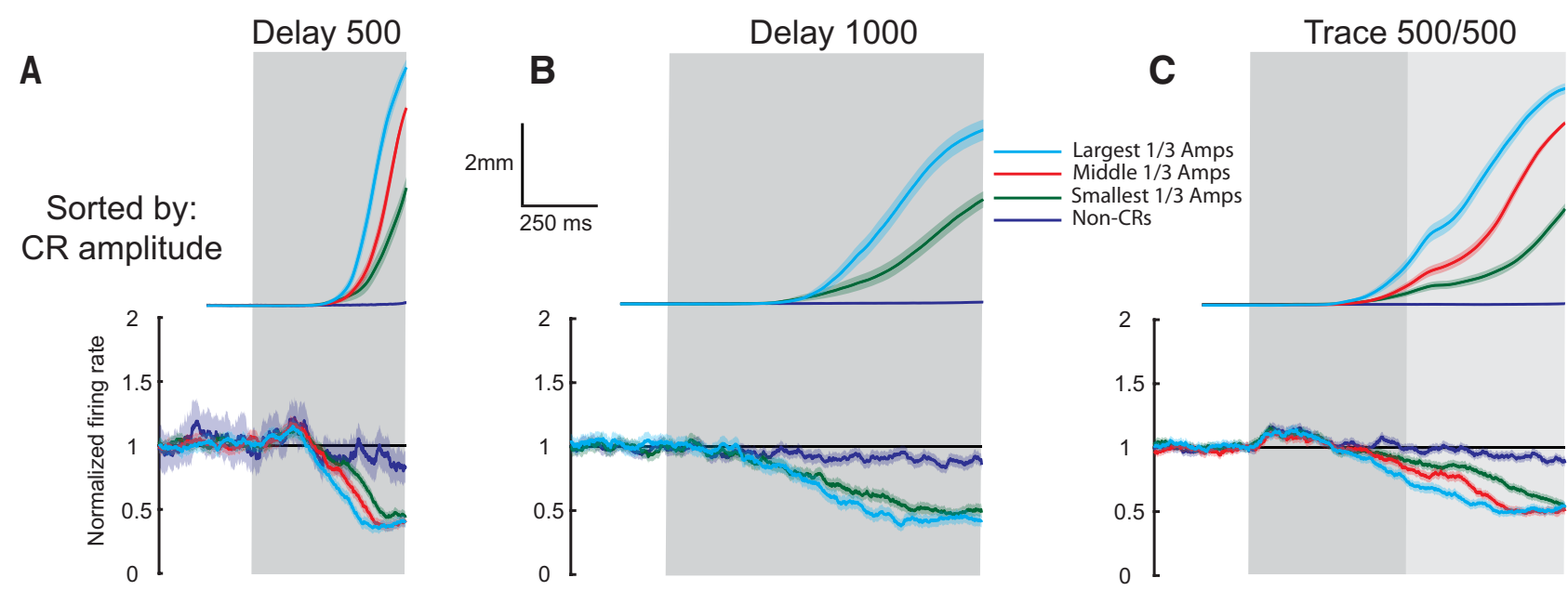

D

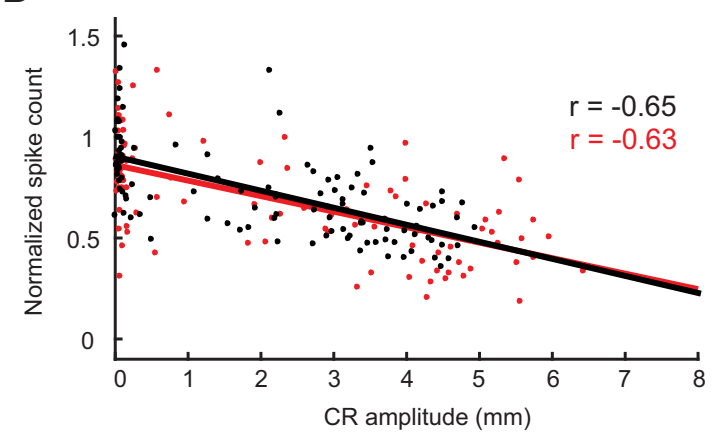

$\mathbf{F}$

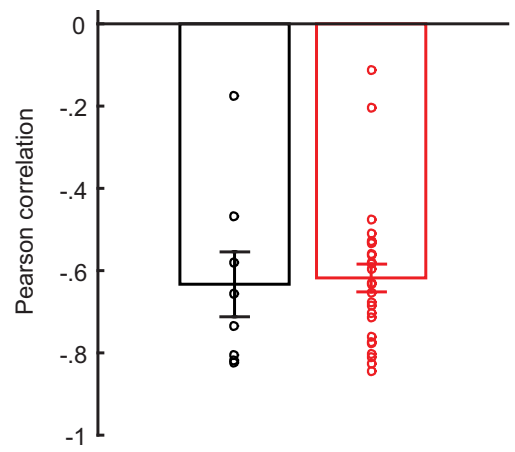

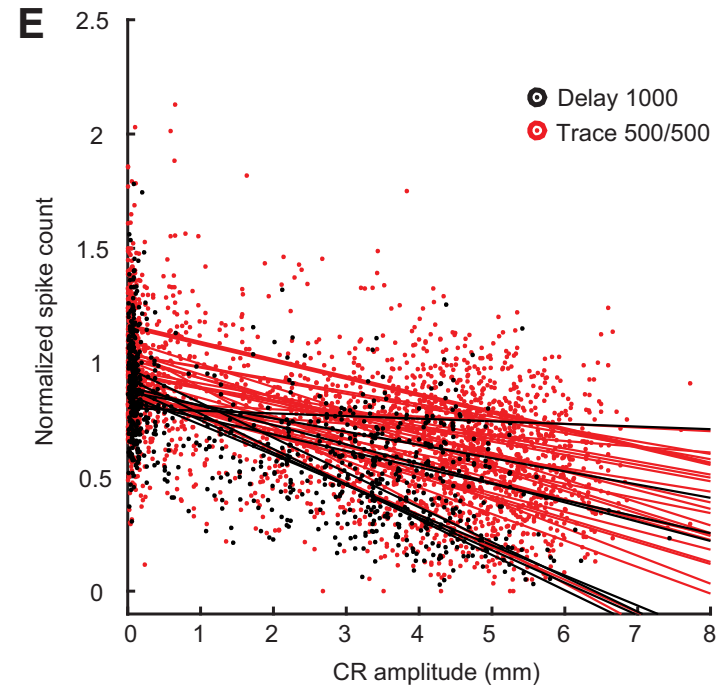

G

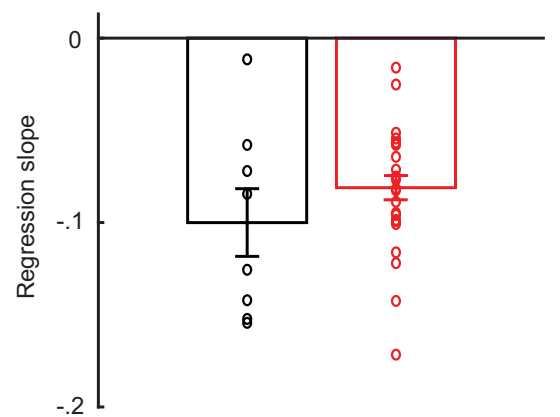

Figure 4. $\quad A-C$, Behavioral CRs and eyelid PC firing rate profiles sorted into groups according to CR amplitude and averaged within each ISI. CRs (top) and eyelid PC data (bottom) sorted by maximum CR amplitude (cyan = largest amplitude, red $=$ middle amplitude, green $=$ smallest amplitude, blue $=$ non-CRs). The dark gray bars represent duration of the $C S$ and light gray represents the trace interval. D, Trial-by-trial correlation between CR amplitude and spike counts normalized to the baseline from eyelid PCs recorded during Delay 1000 and Trace 500/500. Each dot represents the normalized spike count during the last $500 \mathrm{~ms}$ before the US as a function of CR amplitude on individual trials. Dots and best linear fit line correspond to Delay 1000 (black) and Trace $500 / 500$ (red) individual eyelid PC examples. $\boldsymbol{E}$, Summary of trial-by-trial correlations between normalized spike count and CR amplitude for all eyelid PCs recorded during Delay 1000 and Trace 500/500. F, Distributions of the correlation coefficients between spike count and CR amplitude. Each dot represents a single eyelid PC during Delay 1000 and Trace 500/500. Error bars indicate SEM. $\boldsymbol{G}$, Similar as $\boldsymbol{F}$, but comparing linear regression slopes from analyses in $\boldsymbol{E}$.

sponses of the eyelid PC. Robust (well trained) delay responding was accompanied by robust decreases in PC activity. In the transition session from Delay 500 to Trace 500/500, the extinction of delay CRs paralleled the disappearance of the decrease in PC activity. It is unknown whether the early-latency CRs on the next session after the transition session are due to spontaneous recov- ery of the extinguished delay responses or the beginning of trace responding; either way, the responses were accompanied by decreases in PC activity at the appropriate time. Finally, in the last session (Trace 500/500 well trained), the robust trace CRs showed later onsets relative to the earlier onset timing observed during delay conditioning. The later CR onset timing during trace con- 

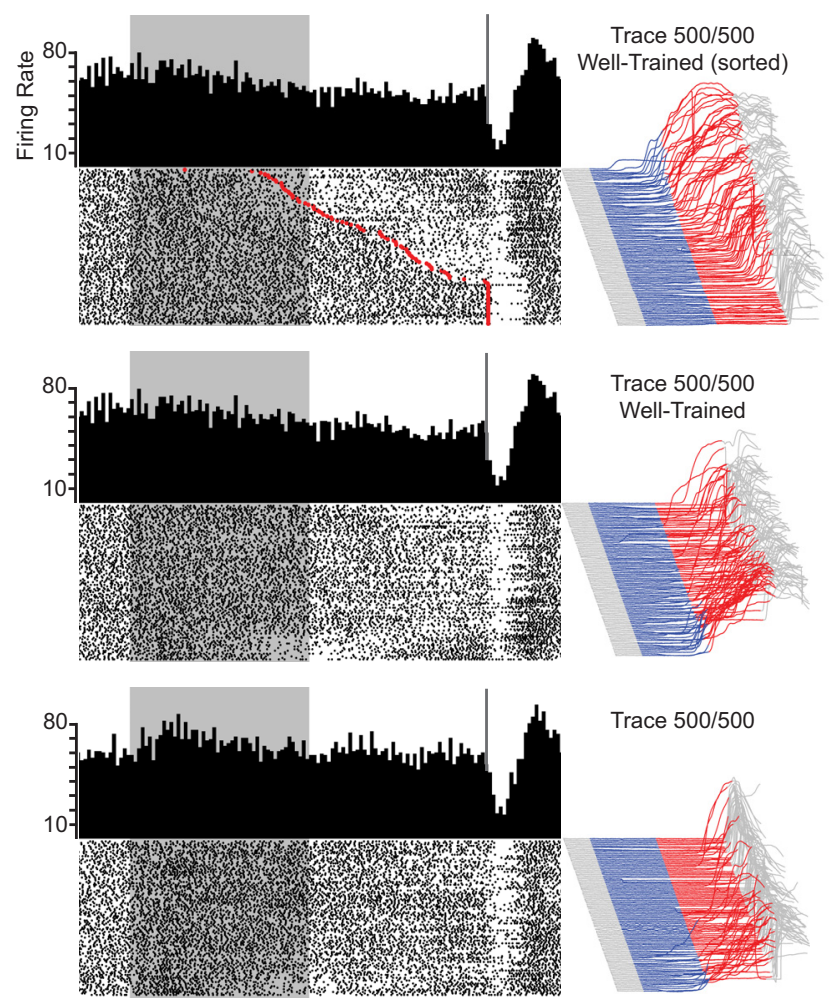

Trace $500 / 500$
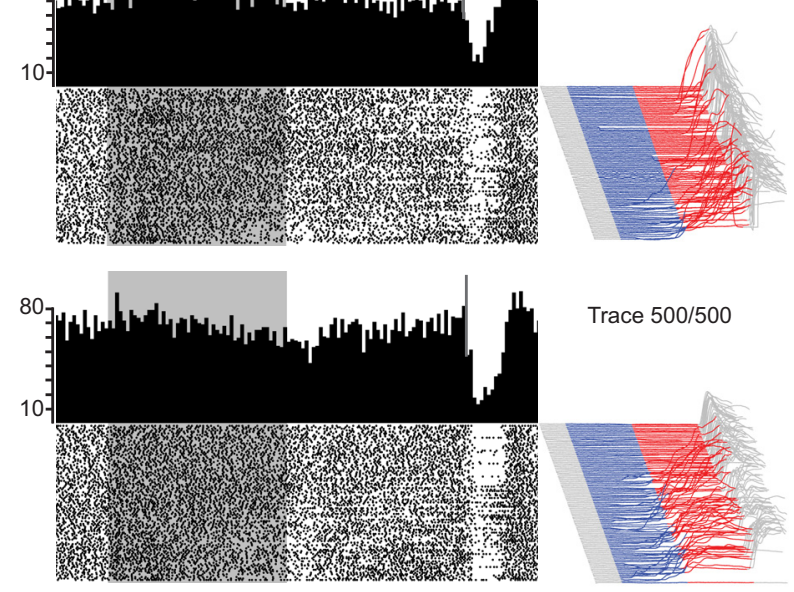

Trace 500/500
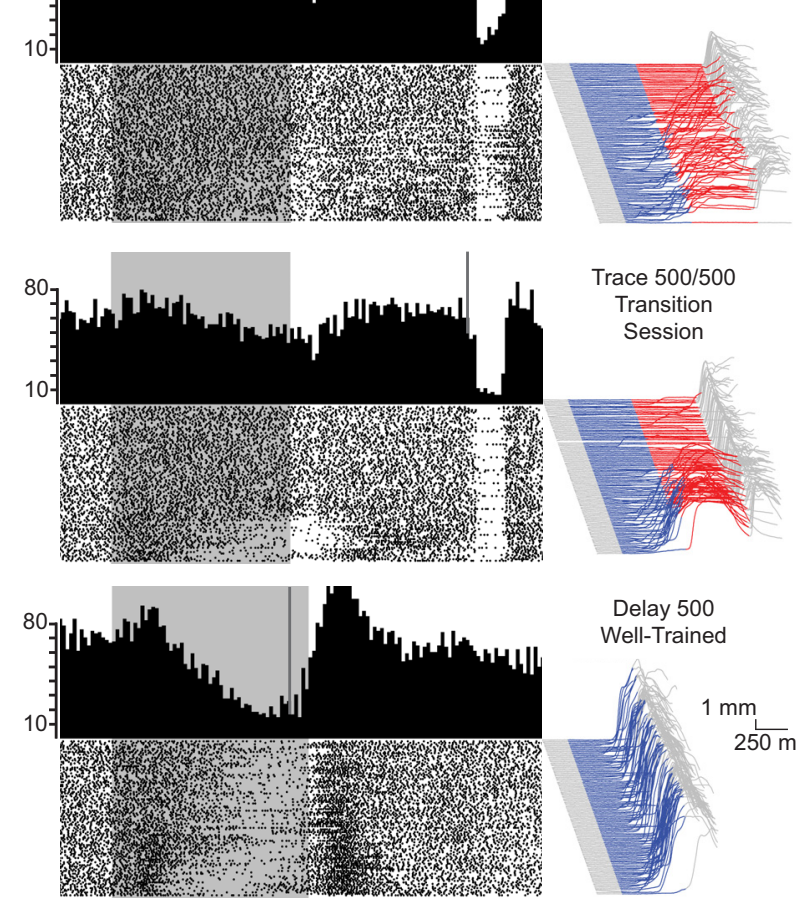

Delay 500 Well-Trained

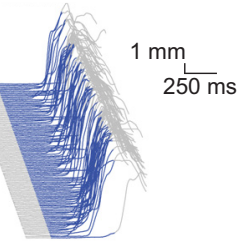

Figure 5. Recordings from a single eyelid $P C$ held throughout the $5 \mathrm{~d}$ required to transition from expression of well trained CRs during Delay 500 (bottom) to Trace 500/500 (top). Peristimulus histograms (10 ms bins) and raster plots in order of each 108 trial session with the corresponding eyelid responses are shown. For the behavioral sweeps, blue represents the duration of the $500 \mathrm{~ms}$ tone $C S$ and red represents the $500 \mathrm{~ms}$ trace interval. Data from the well trained Trace 500/500 session are shown in order of the session and again sorted by CR latency (red dots) (top) to more clearly show the relationship between the decreases in eyelid PC activity and CRs. ditioning was accompanied by correspondingly later decreases in the PC activity. To show more clearly the relationship between decreases in eyelid PC activity and CRs during trace conditioning, the data from the well trained session is also shown sorted by CR onset (Fig. 5, top). This example shows that decreases in activity of a single eyelid PC shifts to match changes in the timing of conditioned eyelid responses expressed during delay and trace conditioning.

In a second instance, we were able to follow a single eyelid PC from expression of Delay 1000 CRs with a $9.5 \mathrm{kHz}$ tone CS through acquisition and robust CR expression during Trace 500/ 500 with a $1 \mathrm{kHz}$ tone CS. Figure $6 A$ shows peristimulus histograms, raster plots, and eyelid responses from four of the eight recording sessions in which this eyelid $\mathrm{PC}$ was followed. The four sessions shown in Figure $6 A$ are, from bottom to top, the first Delay 1000 session, the fourth session of Delay 1000, transition session from Delay 1000 to Trace 500/500 with a different CS, and the fourth session of Trace 500/500 conditioning. To illustrate visually that the relationship between eyelid PC activity and conditioned eyelid responses does not vary for trace versus delay responses, we plotted a combined raster plot of the $\mathrm{PC}$ data from Figure $6 A$ sorted by CR onset with the onsets of Trace 500/500 responses indicated by red dots and the onsets of Delay 1000 responses indicated by blue dots. For both delay and trace responses, decreases in eyelid PC activity occur just before CR onset and non-CR trials for each training paradigm are accompanied by no changes in PC activity (bottom of raster). This PC demonstrated a similar relationship with behavioral responses across both paradigms and shows that a single PC exhibits learningrelated decreases to different CSs.

CRs produced from trace conditioning are better timed (show later latencies to onset and faster rise times) than delay responses with the same ISI (Kalmbach et al., 2010a). Evidence suggests this is mediated by the ability of the cerebellum to learn to respond to persistent inputs and to suppress responding for CS inputs during trace conditioning, which helps delay CR onsets after CS offset (Kalmbach et al., 2009, 2010b). This improvement in timing is apparent in the example transition from Delay 1000 conditioning to Trace 500/500 conditioning shown in Figure 6A both in the behavioral CRs and in the eyelid PC responses. Sorting these data by latency to CR onset shows the improved timing (of CRs and eyelid PC responses) from the transition session from Delay 1000 to Trace 500/500 conditioning (Fig. $7 B$ ) to the well trained trace conditioning session (Fig. $7 A$ ). To quantify this change in $\mathrm{CR}$ timing, we calculated separate cumulative probability distributions of CR onset times for the two delay sessions versus the two trace sessions from the data shown in Figure 6 (Fig. 7C). A two-sample Kolmogorov-Smirnov test revealed that these distributions were significantly different $(p<0.0001, \alpha=0.01)$. These results demonstrate that this behavioral paradigm produces a genuine transition from Delay 1000 responding, with its characteristic poorer timing, to Trace 500/500 responding, where CRs are more precisely timed at the same overall ISI. The sorted raster plots in Figure 7, $A$ and $B$, suggest qualitatively that, despite the change in CR timing, the relationship between eyelid PC responses and CRs does not change, consistent with the hypothesis that eyelid PC activity controls CR expression similarly for trace and for delay conditioning.

Inverse model predictions of PC activity during delay and trace conditioning

We have demonstrated above that the relationship between learning-related decreases in eyelid PCs and the onset of CRs and 
A
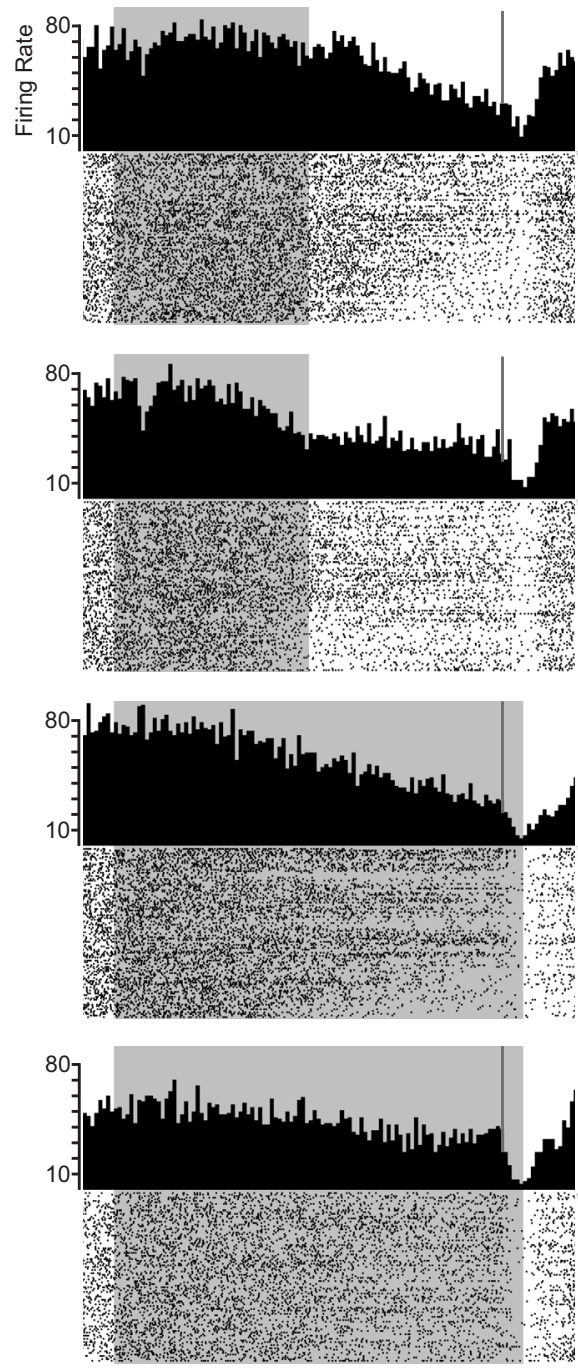

\section{Trace 500/500}

Well-Trained

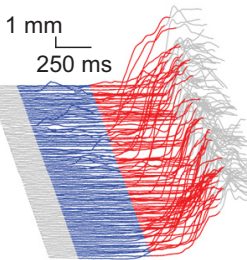

Trace 500/500

Transition

Session

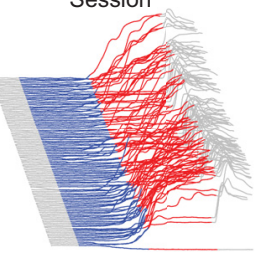

Delay 1000

Well-Trained

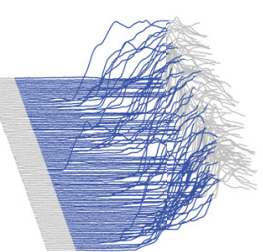

Delay 1000

Well-Trained

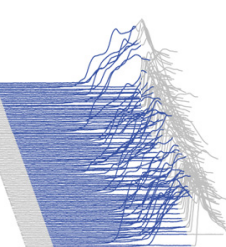

B

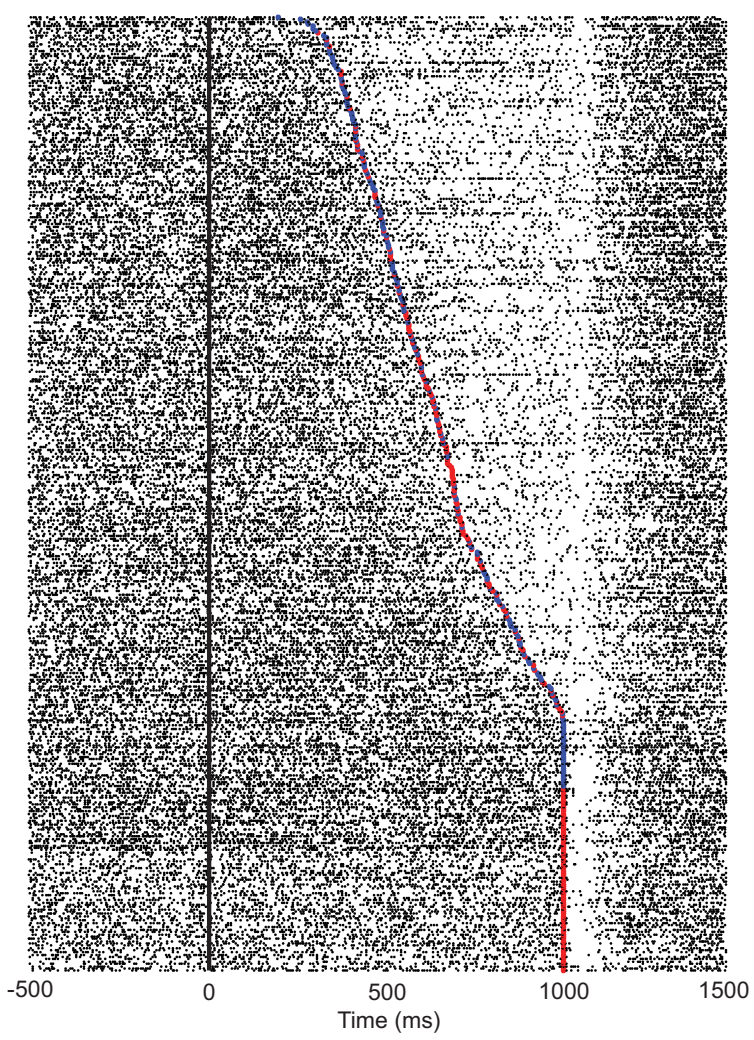

Figure 6. A, Example responses from the single eyelid PC that was recorded throughout the transition from expression of well trained CRs with Delay 1000 to expression of CRs with Trace $500 / 500$ (and a different tone (S). Peristimulus histograms (10 ms bins), raster plots, and eyelid responses are shown in chronological order (bottom to top) from two Delay 1000 and two Trace $500 / 500$ sessions. The behavioral sweeps show how the timing of the CRs changed throughout the transition from delay to trace conditioning. Blue represents duration of the tone CS and red represents the duration of the trace interval. Note the close trial-to-trial correspondence between the decreases in eyelid PC activity and CR onset in each session. $\boldsymbol{B}$, All trials from the sessions shown in $\boldsymbol{A}$ were combined into a single raster plot and sorted by CR onset. This was done to once again demonstrate the consistent relationship between eyelid PC responses and eyelid CRs during both delay and trace eyelid conditioning. Trials with CR onset indicated with blue dots represent Delay 1000 trials and red dots represent Trace 500/500 trials. The black line at time $=0$ represents CS onset.

the correlation between CR amplitude and PC spike count are consistent across both delay and trace conditioning. Among the strongest evidence that learning-related changes in PCs control the expression of conditioned eyelid responses (in delay conditioning) is the tight relationship between eyelid PC activity and the kinematic properties of CRs. The relationship, or lack thereof, between eyelid PC activity and trace responses represents a strong test of how similar or different the cerebellar cortex contributions are to delay versus trace CRs. This makes the question whether the relationships between the temporal firing rate profile of eyelid PCs and the kinematics of the CRs as a function of time are consistent across delay and trace paradigms of central importance. We tested this question using an inverse model approach previously used to model the relationship between PC activity and behavior in smooth pursuit studies (Medina and Lisberger, 2007, 2009). In the inverse model, the PC firing rate profile is modeled as a linear weighed sum of eyelid position, velocity, and acceleration as a function of time in addition to a baseline rate constant. The weights in the model ( $a-c$ in the equation) relate to the relative contributions of position, velocity, and acceleration to the firing rate time profile.

Data used to fit the model and data used to predict PC firing rate from the model were each based on separate sets of trials (see Materials and Methods). The accuracy of the model was determined by the amount of variance of the actual average eyelid PC firing rate on test trials that was captured by the prediction. Figure 8 shows the inverse model applied to each eyelid PC recording for Delay $1000(n=8)$ and Trace 500/500 $(n=26)$. Examples of individual eyelid PC recordings during Delay 1000 (Fig. 8A) and Trace 500/500 (Fig. $8 B$ ) show the modeling results for the two example individual units. Average actual eyelid PC firing rate (brown lines) from different samples of test trials from the session, along with the model's predicted PC firing rate (orange lines), are shown at the top. The amount of variance of the actual PC firing rate captured by the prediction is shown for every PC recorded during Delay 1000 and Trace 500/500 in Figure 8C. The 
A

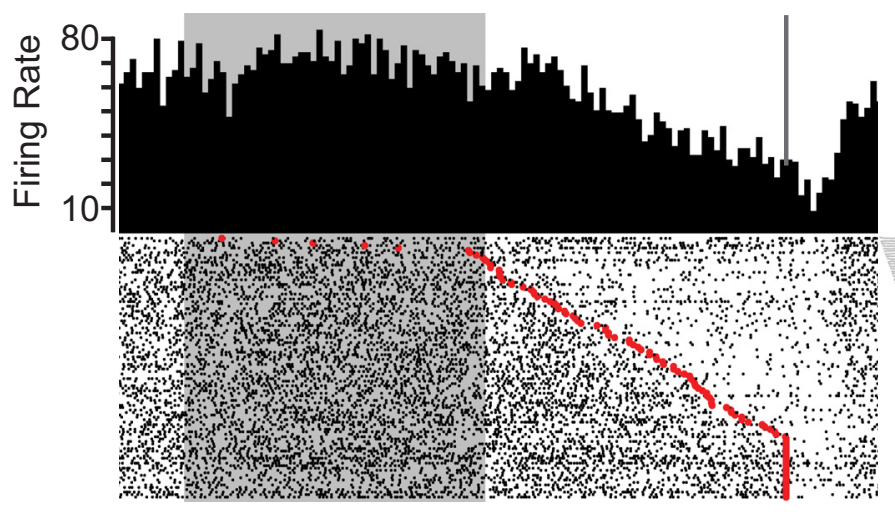

B

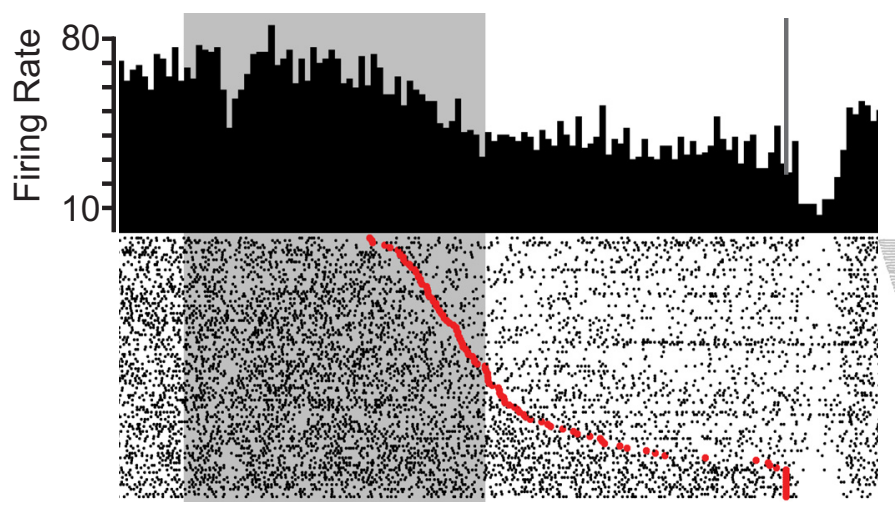

Trace 500/500

Well-Trained

(sorted)

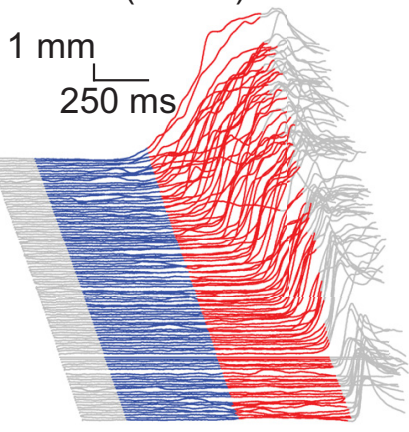

Trace 500/500

Transition Session

(sorted)

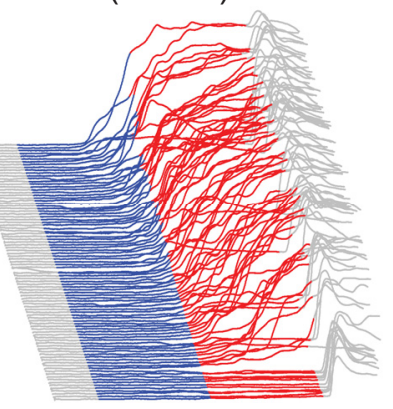

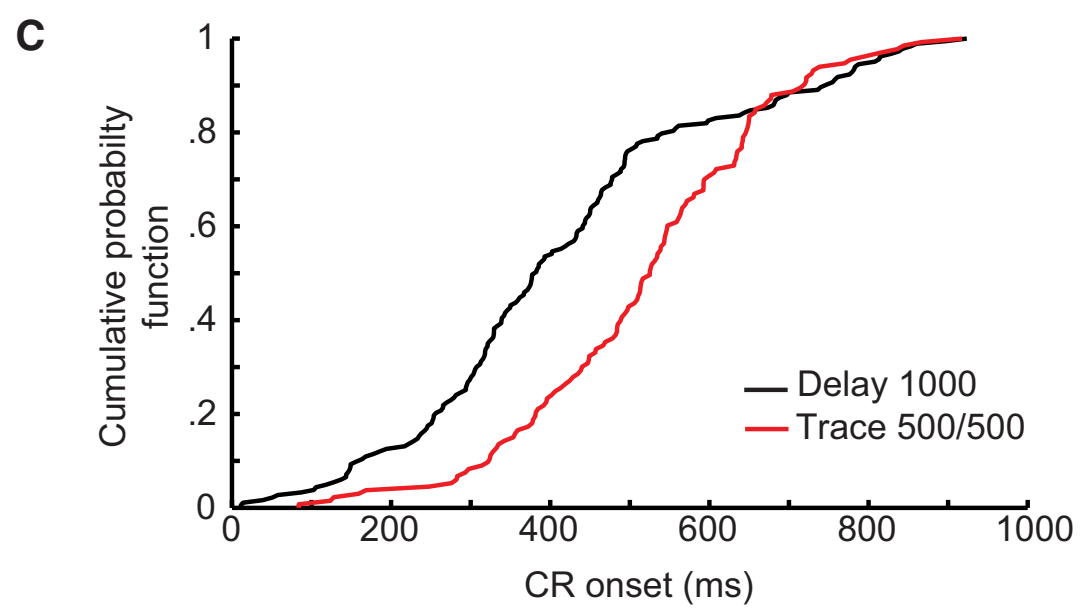

Figure 7. $\quad \boldsymbol{A}, \boldsymbol{B}$, Peristimulus histograms ( $10 \mathrm{~ms}$ bins), raster plots and eyelid CRs sorted by CR onset (red dots) are shown for the transition $(\boldsymbol{B})$ and well trained $(\boldsymbol{A})$ Trace $500 / 500$ sessions from Figure 6. These two sessions illustrate the improvement in CR onset latency (timing) during the switch from Delay 1000 to Trace 500/500. Note the number of CRs that begin during the CS on the transition session of trace conditioning, whereas very few CRs begin during the CS during the well trained session. C, Cumulative probability distributions of CR onset latency for the Delay 1000 (black) and Trace 500/500 (red) sessions from Figure 6 illustrating the later onset latency during Trace 500/500 conditioning relative to Delay 1000 at the same overall ISI.

inverse model provides accurate predictions of eyelid PC responses for both Delay $1000\left(R^{2}=0.66 \pm 0.08\right)$ and Trace 500/ $500\left(R^{2}=0.60 \pm 0.04\right)$. Because the number of test trials used to generate each prediction was low (mean $=20$ per iteration), the variance not captured by the model likely comes from random firing rate fluctuations around the mean baseline rate from trial to trial. Prediction accuracy was not significantly different across eyelid PC populations from delay and trace paradigms (twosample bootstrap test, $p=0.23,10,000$ repetitions).
The eyelid PC firing rate prediction by the inverse model is based on the linear combination of eyelid position, velocity, and acceleration time profiles. The eyelid position, velocity, and acceleration contributions are shown at the bottom of Figure 8, $A$ and $B$, for Delay 1000 and Trace 500/500, respectively. In each case, thin lines correspond to different samples of test trials and the thicker line represents the average across each of the samples. For both example PCs from delay and trace sessions, eyelid position and velocity provide a similar contribution to the predicted 


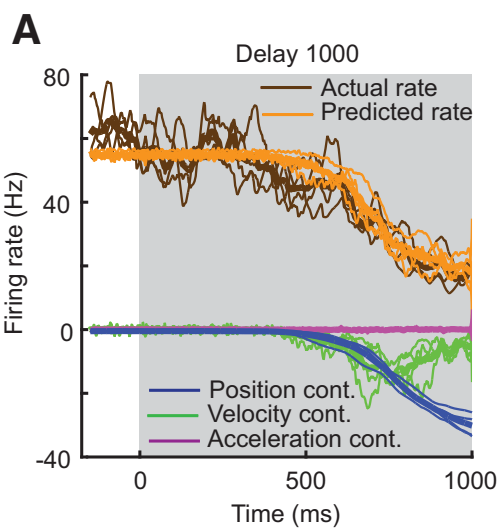

D

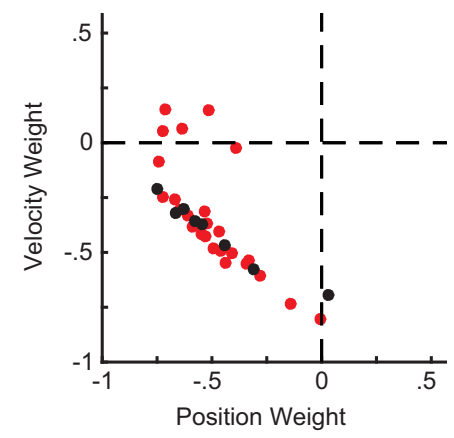

G

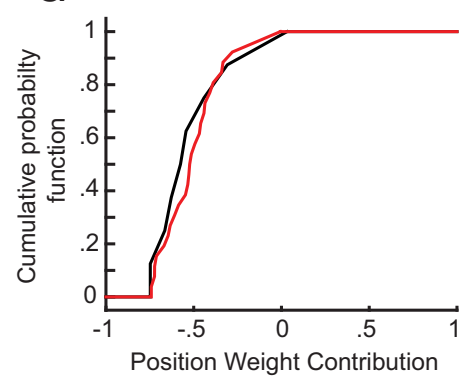

B

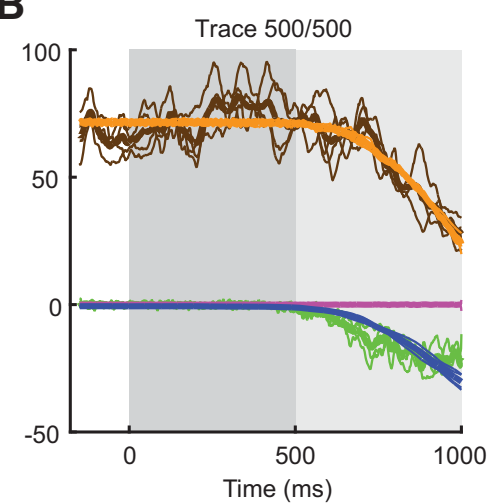

E

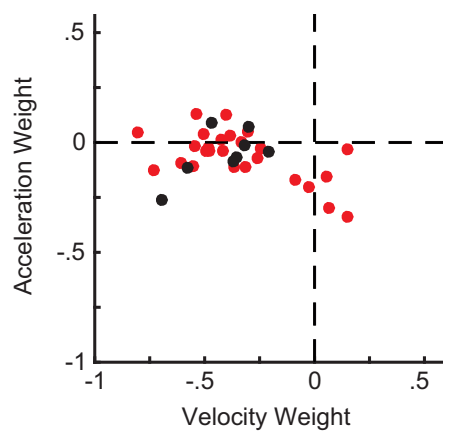

H

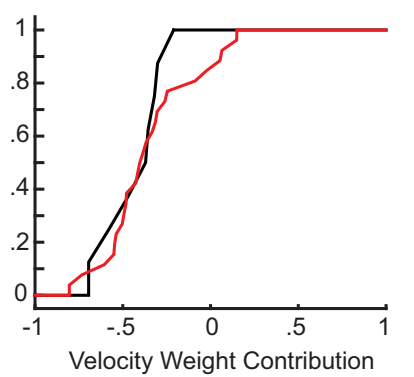

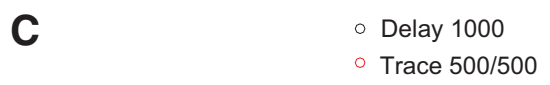

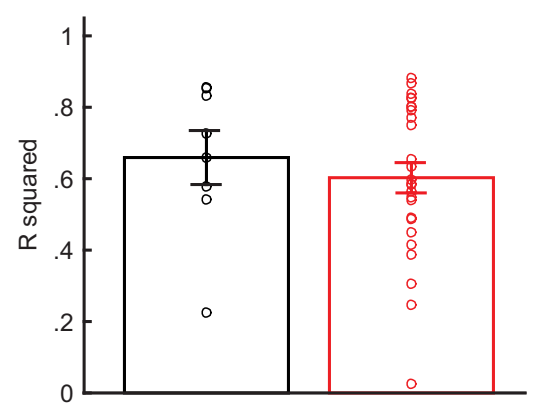

F

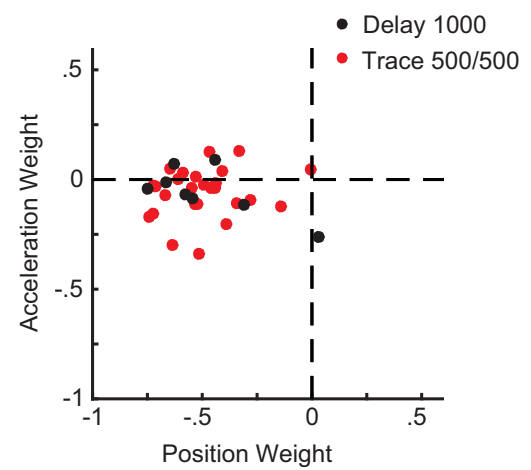

I

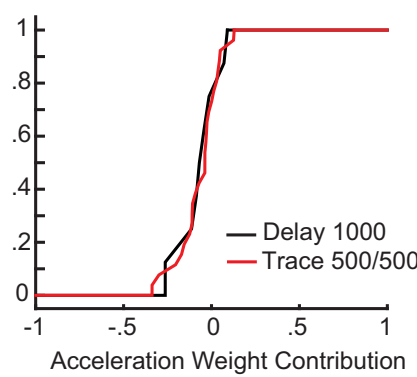

Figure 8. Inverse model analysis of eyelid PC activity during Delay 1000 and Trace 500/500. $A, B$, Examples from individual eyelid PCs of the inverse firing rate model for Delay $1000(\boldsymbol{A})$ and Trace $500 / 500(B)$. Average $P($ firing rate profile (brown lines) on four iterations of test trials (see Materials and Methods), along with predicted firing rate from the inverse model (orange lines). Thin lines represent results from four iterations of test trials and thick lines represent the average across them. Contributions from eyelid position, velocity, and acceleration to the predicted PC firing rate are plotted at the bottom. Dark gray shaded region represents duration of the tone stimulus and light gray represents the trace interval. $C$, Amount of variance of actual PCs firing rate time profile captured by the inverse model. Each dot represents a single eyelid PC color coded according to the training paradigm. Bar plot shows the average $R^{2}$ value across all $\mathrm{PC}$ recordings within the paradigm. Error bars indicate SEM. $\boldsymbol{D}-\boldsymbol{F}$, Relationship among position, velocity, and acceleration weights in the inverse model. Each dot represents a single eyelid $\mathrm{PC}$ color coded according to the training paradigm. G-I, Cumulative probability distributions of each kinematic variable weights in the inverse model plotted separately for each training paradigm.

firing rate, whereas the acceleration component provides a negligible contribution. How the corresponding weights are distributed across eyelid PC populations for both Delay 1000 and Trace $500 / 500$ is shown in Figure 8, D-F. The weights of position, velocity, and acceleration were normalized so that the sum of their absolute values is equal to one. This normalization allowed for investigation of possible differences between how eyelid PCs encode the kinematics of delay and trace CRs at the same interval. The scatter plots (Fig. 8D-F) show that, during both delay and trace conditioning, eyelid position and velocity contributions are dominant, whereas eyelid acceleration weights are nominal (Fig. $8 D-F$, all points are scattered around the $x$-axis for both delay and trace paradigms). The cumulative probability distributions of each kinematic variable weight across all recorded eyelid PCs during Delay 1000 and Trace 500/500 paradigms are shown in Figure 8, G-I. Although there are variations across individual eyelid PCs position and velocity contributions, there were no significant differences in weights of the cumulative distribution function between delay and trace conditioning (two-sample Kolmogorov-Smirnov test, $p=0.82, p=0.85$, and $p=0.94$ for Fig. $8, G-I$, respectively). These results indicate that, not only do eyelid PCs control the kinematics of delay and trace CRs timed to the same interval (ISI 1000), but the way in which those kinematics are related to PC activity is indistinguishable across the two training paradigms.

\section{Inverse model predicts PC activity across delay and trace conditioning}

The ability to accurately predict eyelid PC activity during delay and trace conditioning based on an inverse firing rate model provides the foundation for especially strong and direct comparisons of cerebellar cortex contributions to delay and trace eyelid conditioning. If the relationship between eyelid PC activity and CRs is the same between delay and trace conditioning, then it 
A
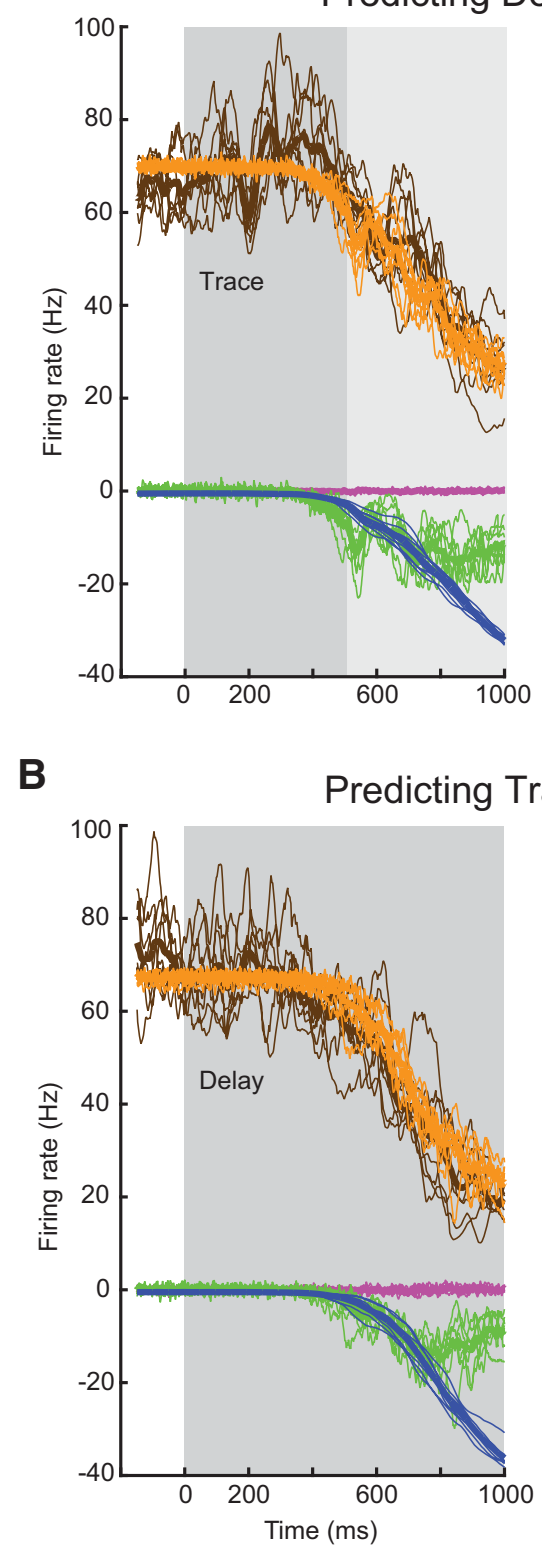

B

C

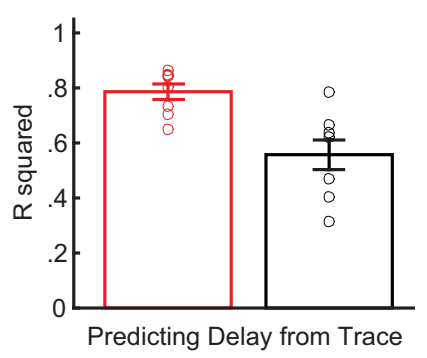

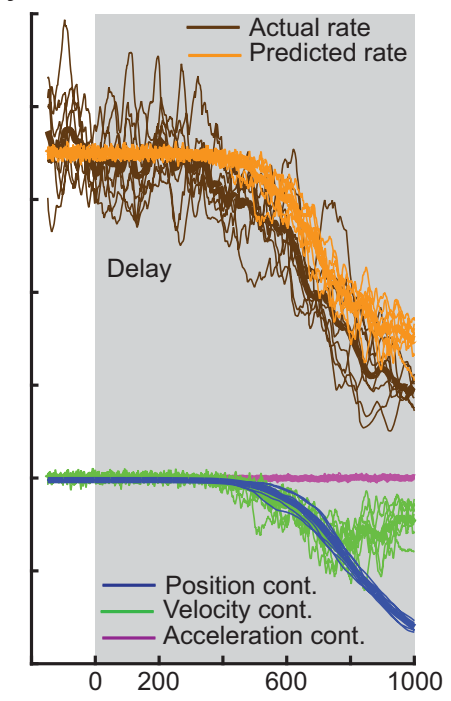

Trace from Delay

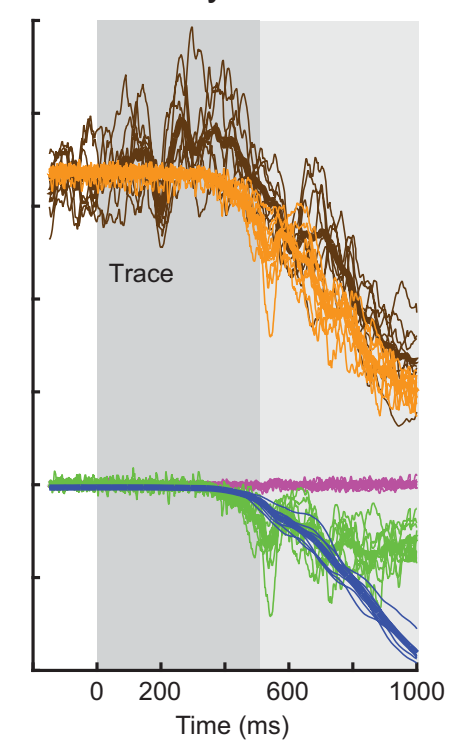

D

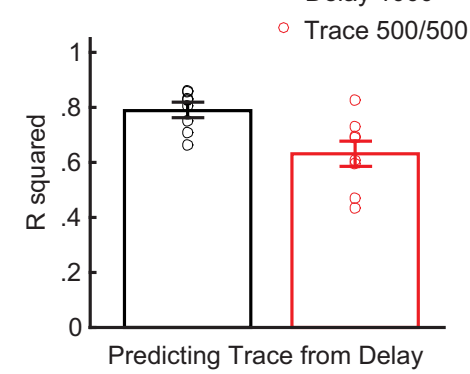

Figure 9. The inverse model was used to make both within- and across-paradigm predictions of eyelid PC activity. All data used for this analysis came from sessions shown in Figure 6.A, PC activity and eyelid kinematics collected during Trace 500/500 were used make an inverse model fit. The results of each fit were applied to make a prediction of PC firing during test trials for both Trace 500/500 (within paradigm) and Delay 1000 (across-paradigm) sessions. Color coding is the same as in Figure 8. Both paradigms are plotted showing actual (brown lines) and predicted (orange lines) PC firing rates averaged across test trials. $\boldsymbol{B}$, Same as $\boldsymbol{A}$, but data from Delay 1000 sessions were used to make an inverse model fit and then make both within- and across-paradigm predictions. $\boldsymbol{C}, \boldsymbol{D}$, Amount of variance of the actual eyelid PC firing rate time profile captured by the inverse model. In each case, the first bar represents the within-paradigm prediction accuracy (e.g., trace predicting trace) and the second bar shows the across-paradigm prediction results (e.g., trace predicting delay). Here, each dot represents the $R^{2}$ value for each test trial iteration and the bar plot shows $R^{2}$ averaged across iterations. Error bars indicate SEM.

should be possible to obtain weights derived from test trials during delay conditioning to then predict eyelid PC activity during trace conditioning (and using trace weights to predict delay) with the same firing rate model. We implemented this test for the eyelid PC shown in Figure 6. We believe these data are from the same eyelid PC held over many sessions, but this assumption is not necessary to make the points below.

Following this logic, we first used a portion of trials from one paradigm (Trace 500/500 for example) to make a model fit and then applied the results of the fit to the test trials within the same paradigm (Fig. 9A, left) or across paradigm to Delay 1000 (Fig. 9A, right). Brown lines in Figure $9 A$ correspond to the actual average $\mathrm{PC}$ firing rate on test trials, thin lines correspond to each iteration of test trials sampled, and the thicker line represents the average. Orange lines are inverse model predictions from the weights obtained from fit data for position, velocity, and acceleration plus a baseline constant. Figure $9 B$ shows the same procedure using the inverse model to predict PC activity during Trace 500/ 500 conditioning from the weights obtained from Delay 1000 conditioning sessions. Across-paradigm predictions of $\mathrm{PC}$ firing rate not only replicated the general time course of a learned decrease in activity, but also captured a large amount of the actual firing rate variance $\left(R^{2}=\right.$ $0.56 \pm 0.05$ for delay from trace, Fig. $9 C$, black bar; $R^{2}=0.63 \pm 0.05$ for trace from delay, Fig. $9 D$, red bar). The ability of the model to reproduce much of the PC data across paradigms is especially compelling given the subtle kinematic differences observed during CR expression of Delay 1000 and Trace 500/500. There was a significant difference in the amount of variance captured by the model when predictions were made across paradigms (two-sample bootstrap test, $p<0.001$, black vs red bars in Fig. $9 C, D)$. The acrossparadigm difference was likely due to two factors. First, as detailed above, the main difference between Delay 1000 and Trace 500/500 comes from CR kinematics, which were used to create the predictions using the inverse model approach. Second, the model was able to account for a large amount of variance for the withinparadigm predictions in both cases. Therefore, the across-paradigm difference in prediction accuracy is likely a reflection of the high accuracy of the withinparadigm predictions combined with the subtle kinematic differences between the 
two paradigms and not the relationship of PC activity to CRs. These results provide additional evidence in support of the general findings indicating that the relationship between eyelid PC firing rate and $\mathrm{CR}$ kinematics is the same for delay and trace conditioning.

\section{Discussion}

We tested the hypothesis, suggested from certain lesion and knock-out studies, that the cerebellar cortex is either not involved with trace eyelid conditioning or, at the very least, that cerebellar cortex involvement is fundamentally different between delay and trace eyelid conditioning (Woodruff-Pak et al., 1985; Kishimoto et al., 2001a,b,c; Takehara-Nishiuchi, 2018). To test this hypothesis, we recorded PC responses from the region of cerebellar cortex known to be necessary for acquisition and properly timed expression of CRs during delay and trace conditioning (Perrett et al., 1993; Garcia et al., 1999; Kalmbach et al., 2010a; Halverson et al., 2015a). We found that eyelid PC responses for both delay and trace CRs were consistent with the hypothesis that the same PCs, or at least PCs in the same small region of cerebellar cortex, control the expression of CRs during both delay and trace conditioning. The relationship between changes in eyelid PC activity and the behavioral expression of eyelid CRs is almost indistinguishable across the two paradigms. These data are inconsistent with the hypotheses that the region of cerebellar cortex involved with expression of delay CRs is not playing the same role during trace eyelid conditioning and that the cerebellar cortex is necessary for delay but not for trace conditioning. Instead, these data support the hypothesis that learning-related changes in PCs control the expression of both trace and delay eyelid CRs and the rules operating in the cerebellar cortex, including whether it is necessary for learning and expression of CRs, do not differ for trace and delay conditioning. Combined with previous results (Kalmbach et al., 2009, 2010b), the present results suggest that the main difference for the cerebellum between delay and trace eyelid conditioning is the complexity of the mossy fiber inputs. In delay conditioning with an auditory CS, the necessary CS input is driven by the medial auditory thalamus projection to pons ( $\mathrm{Hal}-$ verson and Freeman 2006, 2010; Halverson et al., 2008, 2010, 2015b), whereas in trace eyelid conditioning, there is an additional input conveying persistent responses to pons via forebrain or thalamic structures. Although this difference can account for the better timing seen in trace CRs, it does not require fundamentally different cerebellar processes or learning rules.

One subtle difference was apparent in the eyelid PC responses for Delay 1000 versus both Delay 500 and Trace 500/500. In Delay 500 and Trace 500/500, where CR velocities are somewhat higher, there was a small increase in PC activity just after CS onset and before CR onset (Fig. 2C). This increase was not seen in eyelid PC responses during Delay 1000. Previous work involving both empirical data and simulations of the cerebellum suggested that increases in PC activity early in the CS may improve timing by actively suppressing activity in the deep cerebellar nuclei (Medina et al., 2000). The absence of an initial increase in eyelid PC activity during Delay 1000 may relate to the poorer timing observed when rabbits are trained at longer ISIs relative to Delay 500 and Trace 500/500 (Kalmbach et al., 2010b).

The initial suggestion that cerebellar cortex is not necessary for trace conditioning originated from an experiment showing large aspiration lesions of cerebellar cortex had no effect on CR expression during trace conditioning (Woodruff-Pak et al., 1985). Early on, similar conclusions were drawn for delay eyelid conditioning based on studies reporting that lesions of cerebellar cortex failed to produce shifts in CR latency or completely abolished CRs (Yeo et al., 1984, 1985a; Lavond and Steinmetz 1989; Yeo and Hardimann 1992; Clark and Lavond 1994; Attwell et al., 1999). Later, lesion and inactivation studies revealed a region of cerebellar cortex more lateral and ventral (between HVI and HV of the anterior lobe) that is necessary for properly timed expression of CRs for both delay and trace conditioning and that is necessary for acquisition and extinction of eyelid CRs for delay conditioning (Perrett et al., 1993; Perrett and Mauk 1995; Garcia and Mauk 1998; Garcia et al., 1999; Kalmbach et al., 2010a). The cerebellum is also sufficient to learn eyelid CRs in a trace protocol when one mossy fiber input is stimulated for the duration of a CS and the stimulation of a separate mossy fiber input persists into the trace interval (Kalmbach et al., 2010b). Therefore, for both delay and trace conditioning, early lesion experiments that failed to find effects on timing, acquisition, and extinction may have simply missed the critical region of cerebellar cortex (Nores et al., 2000).

The ability to infer cerebellar processes and function from eyelid conditioning studies, including the necessity of the cerebellar cortex, is predicated on the assumption that CRs are driven solely by cerebellar output. In rabbit, a wide variety of convergent evidence supports this assumption (McCormick and Thompson 1984; Lavond et al., 1984; Yeo et al., 1985b; Berthier and Moore 1990; Krupa et al., 1993; Krupa and Thompson 1997; Garcia and Mauk 1998; Choi and Moore 2003; Kalmbach et al., 2010a; Halverson et al., 2015a). The status of this assumption for mouse is considerably less certain. Although Heiney et al. (2014) demonstrated that mouse delay eyelid CRs are cerebellar dependent under particular training conditions, other recent studies have demonstrated that mouse eyelid CRs can be driven by noncerebellar processes (Boele et al., 2010; Sakamoto and Endo 2010; Schonewille et al., 2011; Siegel et al., 2012). To the extent that this is true, demonstrations of spared learning in mice with degenerating PCs or with deficits in LTD at synapses onto PCs (Kishimoto et al., 2001a,b,c; Brown et al., 2010; Yang et al., 2015) may have no bearing on the necessity of these processes in cerebellar learning. Such conclusions require additional controls such as demonstrating that the CRs in these mice are mediated by the cerebellum - that they are, for example, abolished by lesions of cerebellar deep nuclei. In many of these studies, the behavioral attributes of the learning indicate a strong likelihood that the CRs are mediated by noncerebellar processes and even by nonassociative processes.

Delay and trace conditioning differ only with respect to the number of inputs to the cerebellum necessary for learning and expression of CRs. Based on our eyelid PC recordings, the US engages the same eyelid region of inferior olive during both delay and trace conditioning. These climbing fiber inputs then dictate the PCs that will be involved in the learning and execution of the CR for any task (trace or delay) involving eyelid responses. In addition to climbing fiber input, delay conditioning requires mossy fiber input from the CS, whereas trace conditioning requires an additional persistent input to bridge the temporal gap (trace interval) between CS offset and the US (Clark and Squire 1998; Siegel et al., 2012). The cerebellum learns to the persistent input during trace conditioning while at the same time suppressing behavioral responses to the CS (tone) input. This learning mechanism results in CRs during trace conditioning that show better timing (i.e., longer latency to onset) relative to delay CRs trained to the same overall interval (Kalmbach at al., 2011). We propose that, in rabbit, evidence uniformly supports the hypothesis that both delay and trace eyelid conditioning similarly de- 
pend on the cerebellar cortex. Lesions and reversible inactivation of the eyelid region of cerebellar cortex produce comparable effects for both delay and trace eyelid conditioning (Kalmbach at al., 2010a), climbing fiber inputs determine which area of cerebellar cortex controls learning, and the present recordings show that PCs from this region relate in the same ways for trace and delay conditioning to the presence, timing, and amplitude of conditioned responses.

\section{References}

Attwell PJ, Rahman S, Ivarsson M, Yeo CH (1999) Cerebellar cortical AMPA-kainate receptor blockade prevents performance of classically conditioned nictitating membrane responses. J Neurosci 19:RC45. CrossRef Medline

Berger TW, Orr WB (1983) Hippocampectomy selectively disrupts discrimination reversal conditioning of the rabbit nictitating membrane response. Behav Brain Res 8:49-68. CrossRef Medline

Berthier NE, Moore JW (1990) Activity of deep cerebellar nuclear cells during classical conditioning of nictitating membrane extension in rabbits. Exp Brain Res 83:44-54. Medline

Boele HJ, Koekkoek SK, De Zeeuw CI (2010) Cerebellar and extracerebellar involvement in mouse eyeblink conditioning: the ACDC model. Front Cell Neurosci 3:19. CrossRef Medline

Brown KL, Agelan A, Woodruff-Pak DS (2010) Unimpaired trace classical eyeblink conditioning in Purkinje cell degeneration (pcd) mutant mice. Neurobiol Learn Mem 93:303-311. CrossRef Medline

Choi JS, Moore JW (2003) Cerebellar neuronal activity expresses the complex topography of conditioned eyeblink responses. Behav Neurosci 117: 1211-1219. CrossRef Medline

Christian KM, Thompson RF (2003) Neural substrates of eyeblink conditioning: acquisition and retention. Learn Mem 10:427-455. CrossRef Medline

Clark RE, Lavond DG (1994) Reacquisition of eyeblink classical conditioning following large cerebellar cortical lesions in dutch belted rabbits. Behav Brain Res 61:101-106. CrossRef Medline

Clark RE, Squire LR (1998) Classical conditioning and brain systems: the role of awareness. Science 280:77-81. CrossRef Medline

Eccles JC, Ito M, Szentagothai J (1967) The cerebellum as a neuronal machine. New York, NY: Springer.

Ekerot CF, Larson B (1979) The dorsal spino-olivocerebellar system in the cat. I. Functional organization and termination in the anterior lobe. Exp Brain Res 36:201-217. CrossRef Medline

Garcia KS, Mauk MD (1998) Pharmacological analysis of cerebellar contributions to the timing and expression of conditioned eyelid responses. Neuropharmacology 37:471-480. CrossRef Medline

Garcia KS, Steele PM, Mauk MD (1999) Cerebelar cortex lesions prevent acquisition of conditioned eyelid responses. J Neurosci 19:10940-10947. CrossRef Medline

Green JT, Steinmetz JE (2005) Purkinje cell activity in the cerebellar anterior lobe after rabbit eyeblink conditioning. Learn Mem 12:260-269. CrossRef Medline

Groenewegen HJ, Voogd J, Freedman SL (1979) The parasagittal zonation within the olivocerebellar projection. II. climbing fiber distribution in the intermediate and hemispheric parts of cat cerebellum. J Comp Neurol 183:551-601. CrossRef Medline

Halverson HE, Freeman JH (2006) Medial auditory thalamic nuclei are necessary for eyeblink conditioning. Behav Neurosci 120:880-887. CrossRef Medline

Halverson HE, Freeman JH (2010) Medial auditory thalamic input to the lateral pontine nuclei is necessary for auditory eyeblink conditioning. Neurobiol Learn Mem 93:92-98. CrossRef Medline

Halverson HE, Khilkevich A, Mauk MD (2015a) Relating cerebellar purkinje cell activity to the timing and amplitude of conditioned eyelid responses. J Neurosci 35:7813-7832. CrossRef Medline

Halverson HE, Lee I, Freeman JH (2010) Associative plasticity in the medial auditory thalamus and cerebellar interpositus nucleus during eyeblink conditioning. J Neurosci 30:8787-8796. CrossRef Medline

Halverson HE, Poremba A, Freeman JH (2008) Medial auditory thalamus inactivation prevents acquisition and retention of eyeblink conditioning. Learn Mem 15:532-538. CrossRef Medline

Halverson HE, Poremba A, Freeman JH (2015b) Medial auditory thalamus is necessary for acquisition and retention of eyeblink conditioning to cochlear nucleus stimulation. Learn Mem 22:258-266. CrossRef Medline

Heiney SA, Wohl MP, Chettih SN, Ruffolo LI, Medina JF (2014) Cerebellardependent expression of motor learning during eyeblink conditioning in head-fixed mice. J Neurosci 34:14845-14853. CrossRef Medline

Kalmbach BE, Davis T, Ohyama T, Riusech F, Nores WL, Mauk MD (2010a) Cerebellar cortex contributions to the expression and timing of conditioned eyelid responses. J Neurophysiol 103:2039-2049. CrossRef Medline

Kalmbach BE, Ohyama T, Kreider JC, Riusech F, Mauk MD (2009) Interactions between prefrontal cortex and cerebellum revealed by trace eyelid conditioning. Learn Mem 16:86-95. CrossRef Medline

Kalmbach BE, Ohyama T, Mauk MD (2010b) Temporal patterns of inputs to cerebellum necessary and sufficient for trace eyelid conditioning. J Neurophysiol 104:627-640. CrossRef Medline

Kalmbach BE, Voicu H, Ohyama T, Mauk MD (2011) A subtraction mechanism of temporal coding in cerebellar cortex. J Neurosci 31:2025-2034. CrossRef Medline

Kishimoto Y, Hirono M, Sugiyama T, Kawahara S, Nakao K, Kishio M, Katsuki M, Yoshioka T, Kirino Y (2001a) Impaired delay but normal trace eyeblink conditioning in PLCbeta4 mutant mice. Neuroreport 12:2919_ 2922. CrossRef Medline

Kishimoto Y, Kawahara S, Fujimichi R, Mori H, Mishina M, Kirino Y (2001b) Impairment of eyeblink conditioning in GluRdelta2-mutant mice depends on the temporal overlap between conditioned and unconditioned stimuli. Eur J Neurosci 14:1515-1521. CrossRef Medline

Kishimoto Y, Suzuki M, Kawahara S, Kirino Y (2001c) Age-dependent impairment of delay and trace eyeblink conditioning. Neuroreport 12:3349 3352. CrossRef Medline

Krupa DJ, Thompson RF (1997) Reversible inactivation of the cerebellar interpositus nucleus completely prevents acquisition of the classically conditioned eye-blink response. Learn Mem 3:545-556. CrossRef Medline

Krupa DJ, Thompson JK, Thompson RF (1993) Localization of a memory trace in the mammalian brain. Science 260:989-991. CrossRef Medline

Lavond DG, Lincoln JS, McCormick DA, Thompson RF (1984) Effect of bilateral lesions of the dentate and interpositus cerebellar nuclei on conditioning of heart-rate and nictitating membrane/eyelid responses in the rabbit. Brain Res 305:323-330. CrossRef Medline

Lavond DG, Steinmetz JE (1989) Acquisition of classical conditioning without cerebellar cortex. Behav Brain Res 33:113-164. Medline

McCormick DA, Thompson RF (1984) Neuronal responses of the rabbit cerebellum during acquisition and performance of a classically conditioned nictitating membrane-eyelid response. J Neurosci 4:2811-2822. CrossRef Medline

Medina JF, Lisberger SG (2007) Variation, signal, and noise in cerebellar sensory-motor processing for smooth-pursuit eye movement. J Neurosci 27:6832-6842. CrossRef Medline

Medina JF, Lisberger SG (2009) Encoding and decoding of learned smoothpursuit eye movements in the floccular complex of the monkey cerebellum. J Neurophysiol 102:2039-2054. CrossRef Medline

Medina JF, Garcia KS, Nores WL, Taylor NM, Mauk MD (2000) Timing mechanisms in the cerebellum: testing predictions of a large-scale computer simulation. J Neurosci 20:5516-5525. CrossRef Medline

Moyer JR Jr, Deyo RA, Disterhoft JF (1990) Hippocampectomy disrupts trace eye-blink conditioning in rabbits. Behav Neurosci 104:243-252. CrossRef Medline

Nores WL, Medina JF, Steele PM, Mauk MD (2000) Relative contributions of cerebellar cortex and cerebellar nucleus to eyelid conditioning. In: Eyeblink classical conditioning, Vol II: Animal models. (Woodruff-Pak DS, Steinmetz JE, eds), pp 205-228. Boston, MA: Kluwer.

Ohyama T, Nores WL, Medina JF, Riusech FA, Mauk MD (2006) Learninginduced plasticity in deep cerebellar nucleus. J Neurosci 26:12656-12663. CrossRef Medline

Perrett SP, Mauk MD (1995) Extinction of conditioned eyelid responses requires the anterior lobe of cerebellar cortex. J Neurosci 15:2074-2080. CrossRef Medline

Perrett SP, Ruiz BP, Mauk MD (1993) Cerebellar cortex lesions disrupt learing-dependent timing of conditioned eyelid responses. J Neurosci 13:1708-1718. CrossRef Medline

Sakamoto T, Endo S (2010) Amygdala, deep cerebellar nuclei and red nu- 
cleus contribute to delay eyeblink conditioning in C57Bl/6 mice. Eur J Neurosci 32:1537-1551. CrossRef Medline

Schonewille M, Gao Z, Boele HJ, Veloz MF, Amerika WE, Simek AA, De Jeu MT, Steinberg JP, Takamiya K, Hoebeek FE, Linden DJ, Huganir RL, De Zeeuw CI (2011) Reevaluating the role of LTD in cerebellar motor learning. Neuron 70:43-50. CrossRef Medline

Siegel JJ, Kalmbach B, Chitwood RA, Mauk MD (2012) Persistent activity in a cortical-to-subcortical circuit: bridging the temporal gap in trace eyelid conditioning. J Neurophysiol 107:50-64. CrossRef Medline

Solomon PR, Moore JW (1975) Latent inhibition and stimulus generalization of the classically conditioned nictitating membrane response in rabbits (Oryctolagus cuniculus) following dorsal hippocampal ablation. J Comp Physiol Psychol 89:1192-1203. CrossRef Medline

Solomon PR, Vander Schaaf ER, Thompson RF, Weisz DJ (1986) Hippocampus and trace conditioning of the rabbit's classically conditioned nictitating membrane response. Behav Neurosci 100:729-744. CrossRef Medline

Takehara-Nishiuchi K (2018) The anatomy and physiology of eyeblink classical conditioning. Curr Top Behav Neurosci 37:297-323. CrossRef Medline

Woodruff-Pak DS, Lavond DG, Thompson RF (1985) Trace conditioning: abolished by cerebellar nuclear lesions but not lateral cerebellar cortex aspirations. Brain Res 348:249-260. CrossRef Medline

Woodruff-Pak DS, Green JT, Levin SI, Meisler MH (2006) Inactivation of sodium channel Scn8A (Na-sub(v)1.6) in purkinje neurons impairs learning in morris water maze and delay but not trace eyeblink classical conditioning. Behav Neurosci 120:229-240. CrossRef Medline

Yang Y, Lei C, Feng H, Sui JF (2015) The neural circuitry and molecular mechanisms underlying delay and trace eyeblink conditioning in mice. Behav Brain Res 278:307-314. CrossRef Medline

Yeo CH, Hardiman MJ (1992) Cerebellar cortex and eyeblink conditioning: a reexamination. Exp Brain Res 88:623-638. Medline

Yeo CH, Hardiman MJ, Glickstein M (1984) Discrete lesions of the cerebellar cortex abolish the classically conditioned nictitating membrane response of the rabbit. Behav Brain Res 13:261-266. CrossRef Medline

Yeo CH, Hardiman MJ, Glickstein M (1985a) Classical conditioning of the nictitating membrane response of the rabbit. II. Lesions of the cerebellar cortex. Exp Brain Res 60:99-113. CrossRef Medline

Yeo CH, Hardiman MJ, Glickstein M (1985b) Classical conditioning of the nictitating membrane response of the rabbit. I. Lesions of the cerebellar nuclei. Exp Brain Res 60:87-98. CrossRef Medline 